\title{
Enhancing Cellular Coverage Quality by Virtual Access Point and Wireless Power Transfer
}

\author{
Jinsong Gui $\mathbb{D}^{1},{ }^{1}$ Lihuan Hui, ${ }^{1}$ and Naixue Xiong $\mathbb{D}^{2}$ \\ ${ }^{1}$ School of Information Science and Engineering, Central South University, Changsha 410083, China \\ ${ }^{2}$ Department of Mathematics and Computer Science, Northeastern State University, Tahlequah, OK 74133, USA
}

Correspondence should be addressed to Jinsong Gui; jsgui06@163.com

Received 19 October 2017; Revised 5 March 2018; Accepted 12 March 2018; Published 29 April 2018

Academic Editor: Oscar Esparza

Copyright (C) 2018 Jinsong Gui et al. This is an open access article distributed under the Creative Commons Attribution License, which permits unrestricted use, distribution, and reproduction in any medium, provided the original work is properly cited.

The ultradensification deploying for cellular networks is a direct and effective method for the improvement of network capacity. However, the benefit is achieved at the cost of network infrastructure investment and operating overheads, especially when there is big gap between peak-hour Internet traffic and average one. Therefore, we put forward the concept of virtual cellular coverage area, where wireless terminals with high-end configuration are motivated to enhance cellular coverage quality by both providing RF energy compensation and rewarding free traffic access to Internet. This problem is formulated as the Stackelberg game based on three-party circular decision, where a Macro BS (MBS) acts as the leader to offer a charging power to Energy Transferring Relays (ETRs), and the ETRs and their associating Virtual Access Points (VAPs) act as the followers to make their decisions, respectively. According to the feedback from the followers, the leader may readjust its strategy. The circular decision is repeated until the powers converge. Also, the better response algorithm for each game player is proposed to iteratively achieve the Stackelberg-Nash Equilibrium (SNE). Theoretical analysis proves the convergence of the proposed game scheme, and simulation results demonstrate its effectiveness.

\section{Introduction}

The proliferation of mobile devices, machine-to-machine (M2M) communication [1], and data collection for smart cities $[2,3]$ leads to the massive growth of Internet traffic. The literature [4] forecasts an increase of thousandfold in wireless traffic in 2020 as compared to the figures in 2010, which can be handled by deploying tremendous amount of small cells resulting in ultradense heterogeneous networks. Although dense networks with small cells for peak-load scenarios can meet soaring capacity and coverage demands in future cellular networks $[5,6]$, these benefits are got at the cost of network infrastructure investment and operating overheads, for example, more access points and higher backhaul costs, more complex interference management, and more power consumption.

Peak-hour Internet traffic increases more rapidly than average Internet traffic. It is reported in [7] that, in 2016, Internet traffic in the busiest 60 -minute period in a day increased 51 percent, while average traffic had only 32 percent growth. Moreover, it is predicted by [7] that, busy-hour Internet traffic will increase by a factor of 4.6 between 2016 and 2021, while average Internet traffic will increase by a factor of 3.2 .

By reducing transmission range and optimizing transmitting power $[8,9]$, network capacity and reliability can be obviously improved due to effective control of mutual interference. Therefore, a simple and easy solution is that network operators can deploy Small Base Stations (SBSs) enough to support peak traffic. However, they suffer from underutilization of resources at low load conditions. In fact, due to high costs for deployment and maintenance, network operators are unwilling to seamlessly deploy dense small cells to meet the customer needs in peak hour.

To address the above problems, we put forward the concept of virtual cellular coverage area, which is an agile, costeffective, and energy efficient alternative for small cell deployments and thus helpful for reducing operational expenditure to the network operators due to the large number of small cell deployments. The problem of continuous operation of 
sparsely deployed SBSs can be tackled by providing data services by cellular Virtual Access Points (VAPs) during peak hours, which can adapt well to the temporal and spatial variations in traffic load. It is more appropriate that wireless terminals with high-end configuration act as VAPs.

VAPs are responsible for receiving data of users far away from a macrostation, for example, cellular edge users, and forward these data to this macrostation, which is helpful for reducing the transmitting powers of cellular edge users and thus alleviating interference to the users in the same frequency bands of adjacent cells. Therefore, a network operator will get more access traffic revenue, but VAPs will consume more energy.

In order to compensate for this energy consumption, a network operator may make a Macro BS (MBS) transfer power to VAPs. However, the power conversion efficiency of the Radio Frequency (RF) energy harvesting circuit decreases sharply with the increase of distance. In order to improve the power conversion efficiency, a MBS should select a few nodes to act as Energy Transferring Relays (ETRs), which can forward energy from a MBS to a VAP.

In many practical scenarios, wireless User Equipment (UE) may be rational such that they only care about their own interests instead of the overall one. Without getting the benefits from the operator, they do not act as either VAPs or ETRs. Therefore, in order to motivate UE to act as either VAPs or ETRs, the operator has to provide it with free traffic access to Internet.

To the best of our knowledge, few works have studied how to enhance cellular coverage quality by VAPs and ETRs. This gap actually motivates us to develop a game-theoretic framework for this problem, where any UE is motivated to act as either a VAP or an ETR through providing both RF energy compensation and free traffic access to Internet. The main contributions of this paper are summarized as follows:

(1) We propose a game-based constructing method for virtual cellular coverage area by combining RF energy compensation with free traffic access to Internet, which can make up for gaps of small cell coverage.

(2) We formulate the charging power decision problem and transmission power splitting problem as a Stackelberg game based on three-party circular decision. A reverse induction method is used to analyze this proposed Stackelberg game since it can capture the sequential dependence of the decisions in all the stages of the game.

(3) The new better response strategy of each player is proposed and then the Stackelberg-Nash Equilibrium (SNE) can be achieved by running the proposed algorithms iteratively.

The main notations in this work are listed in Notations Used in Our Work. The rest of the paper is organized as follows. The related works are analyzed and summarized in Section 2. In Section 3, the game scheme for virtual cellular coverage area construction is discussed in detail, including network model, the time-slotted scheme for energy and information transference, the problem formulation and theoretical analysis, and the description for algorithms. The simulation results are given in Section 4 , and the conclusions are drawn in Section 5.

\section{Related Work}

RF energy harvesting sources are usually divided into two categories [10], where the first category belongs to ambient or unintended RF energy harvesting sources and the second one is the type of dedicated or intended RF ones. Also, there are the three main RF energy harvesting structures at receivingends [11], that is, separated structure (i.e., energy harvesting and information decoding circuitry have their own antennas, respectively), time switching structure (i.e., energy harvesting and information decoding circuitry share the same antenna at different time slots), and power splitting structure (i.e., energy harvesting and information decoding circuitry share the same antenna at the same time but split the input power). A time switching structure is usually used more frequently since it is comparatively compact in form factor and relatively effective in harvesting RF energy.

Furthermore, the literature [12] summarizes Simultaneous Wireless Information and Power Transfer (SWIPT) designing schemes which are usually classified as decoupled SWIPT, closed-loop SWIPT, and integrated SWIPT. The decoupled SWIPT is characterized by the information and the power traffic sent from two separate transmitting sources. In closed-loop SWIPT scenarios [13-18], a base station usually powers a wireless device in a downlink and receives the data from the same wireless device in the uplink. In an integrated SWIPT scheme [19], both the information and power are carried by the same source over the same signals.

Concurrent multiple RF source transmission reported in some literatures (e.g., [20,21]) will increase total interference. A harvest-then-transmit protocol allows a receiver to first harvest energy from the downlink broadcast signals and then use the harvested energy to send independent uplink information to the MBS or SBS based on Time Division Multiple Access (TDMA). In order to mitigate total interference, the system presented in [22] employs multiple dedicated RF sources with a harvest-then-transmit protocol to improve the harvested energy and data rate. The achievable data rate can increase by up to $87 \%$ when energy is harvested from up to 5 dedicated RF sources, compared to the case when energy is harvested from one source.

Some works have focused on energy transfer in multihop manner for wireless networks; for example, [23] proposes a method to inject power into the network as flows, where injected power is carried in multihop manner, while [24] explores the optimization of multihop wireless energy transfer in wireless networks.

Some typical noncooperative game models (e.g., Stackelberg game [25] and potential game [26]) are widely used in various network information systems [27-29]. A Stackelberg game consists of leaders and followers that compete with each other for certain resources, where the leaders first act by considering behaviors of the followers, and the followers act subsequently.

An exact potential game can characterize the set of Nash Equilibria (NE), where a potential function can track the changes in the payoff due to the unilateral deviation of a player, and one or more NE points may exist and coincide 


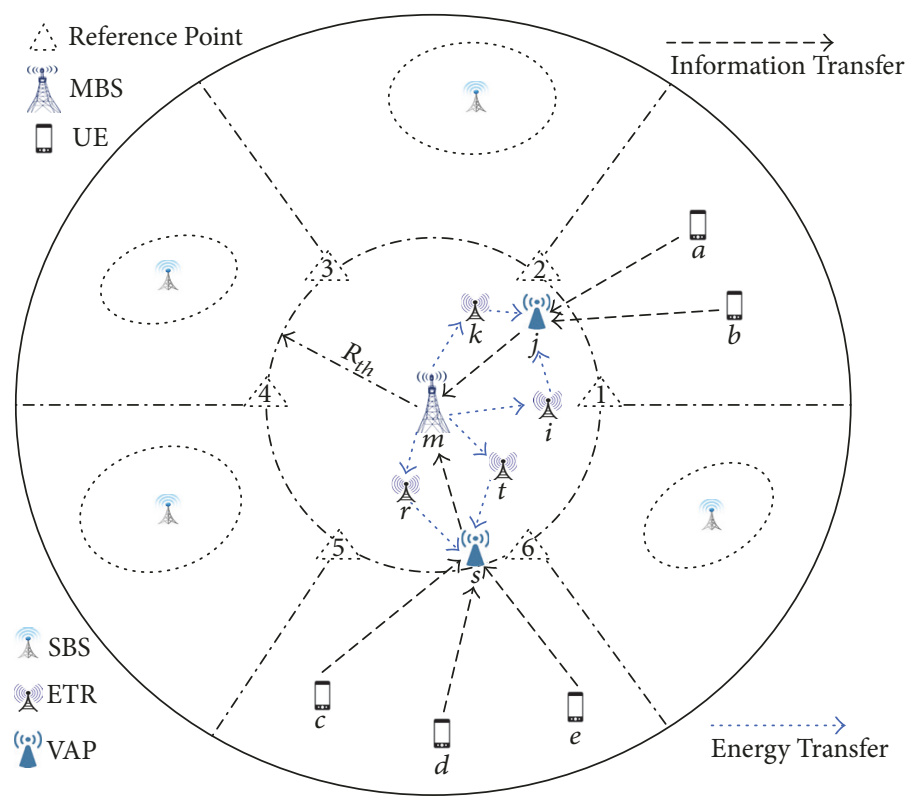

FIGURE 1: Example for heterogeneous cellular networks.

with one point that maximizes the potential function. However, it cannot support application scenarios with sequential dependence.

In RF energy harvesting communication networks, some works [30-35] employ game theory to investigate different setups. However, they hardly have studied the concerned scenario of virtual cellular coverage area construction for a macrocell based on both RF energy compensation and free traffic access to Internet.

In the latest related work [35], the authors consider a radio frequency energy harvesting based Internet of Things (IoT) system, which consists of a data access point (DAP) and several energy access points (EAPs). The DAP collects information from its associated sensors. EAPs can provide wireless charging services to sensors via the RF energy transfer technique.

In this paper, a MBS firstly offers a charging power to the ETRs and then asks each ETR to determine its charging power to its associating VAP, where the purpose is to encourage the VAP to relay data from its associating UE to the MBS by compensating it in terms of energy. Therefore, the Stackelberg game model is applicable to such application scenario with sequential dependence.

\section{The Game Scheme for Virtual Ultra-Network Construction}

3.1. Network Model. We consider a macrocell with a MBS located at its center as illustrated in Figure 1. Also, there are $K$ UE pieces distributed randomly in Figure 1, where each UE may serve as either an ETR or a VAP and also may not do so. To mitigate interference to UE pieces in neighboring macrocells, the maximum transmission power of MBS is usually limited. If the receiving nodes have a strict requirement for the receiving bit error rate, UE pieces far away from a MBS, especially cellular edge users, hardly meet the requirement of such low receiving bit error rate. In addition, as stated in the introduction, even if the cell-edge UE pieces send data to the MBS by using their maximum transmission powers, it is generally difficult for the MBS to achieve such low receiving bit error rate.

There may also be a few SBSs in Figure 1. Therefore, the UE pieces adjacent to the SBSs can access the Internet via the SBSs. However, for the UE pieces far away from SBSs, they may access the Internet via VAPs. Generally, since VAPs do not have backhauls; they forward the data from the cell-edge UE pieces to the MBS.

The hollow triangles in Figure 1 are the reference points used in the election of VAPs, which are usually planned and set by the network operator according to the distribution of the regions without SBSs. Such role as VAP is usually held by the UE pieces that are closer to the reference points and have more energy reserve. However, if the MBS transfers energy to VAPs directly, the power conversion efficiency is usually very low. Therefore, it is necessary to select a few UE pieces to act as ETRs. For example, $i, k, r$, and $t$ in Figure 1 assume such role.

A set of the algorithms for VAP election, ETR election, and UE association is listed in Algorithms 1, 2, and 3, but this paper does not discuss it since we focus on the design and analysis of game scheme for virtual cellular coverage area construction. By Algorithm 1, the MBS (e.g., $m$ in Figure 1) will get its set of VAPs (e.g., $S_{m}=\{j, s$ in Figure 1$\}$ ). Also, any VAP (e.g., $j$ or $s$ in Figure 1) will get its set of ETRs (e.g., $R_{j}$ $=\{i, k$ in Figure 1$\}$ or $R_{s}=\{r, t$ in Figure 1$\left.\}\right)$ by Algorithm 2 , while it will get its set of associated UE pieces (e.g., $U_{j}=\{a, b$ in Figure 1$\}$ or $U_{s}=\{c, d, e$ in Figure 1 $\}$ ) by Algorithm 3 .

3.2. Time-Slotted Scheme for Energy and Information Transference. In general, the MBS adopts OFDMA and communicates with each UE piece over a few subcarriers in 
Run at the macro base station $m$

Input: $S_{m}=\varnothing$

Output: $S_{m}$
(1) Determine the number of virtual access points (e.g., 6)
(2) Set their reference coordinates (e.g., the positions of dotted triangle shown in Figure 1)
(3) For each reference coordinate point (e.g., v) do
(4) Broadcast a request packet at its maximum transmission power
(5) Set $s$ and $d_{s v}$ as 0 and a very large value respectively
(6) Set the timer $t_{\Delta}$ as $\Delta$
(7) while the timer $t_{\Delta}$ does not expire do
(8) If receive a responding packet from node $u$ then
(9) If $d_{s v}>d_{u v}$ then $s=u$ and $d_{s v}=d_{u v}$ End if
(10) End if
(11) End while
(12) Add $s$ to $S_{m}$
(13) Send a confirmation package to node $s$
(14) End for
Run at any node $u$
Input: null
Output: null
(1) If receive a requesting packet with a reference point (e.g., $v)$ then
(2) If $d_{u v}<d_{\mathrm{th}}$ and $e_{u}>e_{\mathrm{th}}$ then
(3) Send a response packet to MBS $m$
(4) End if
(5) End if
(6) If receive a confirmation with a reference point (e.g.,v) then
(7) Mark itself as the virtual access point with respect to the reference coordinate point $v$
(8) End if

Algorithm 1: VAP election.

Run at any virtual access point (VAP) $s \in S_{m}$

Input: $R_{s}=\varnothing$

Output: $R_{s}$

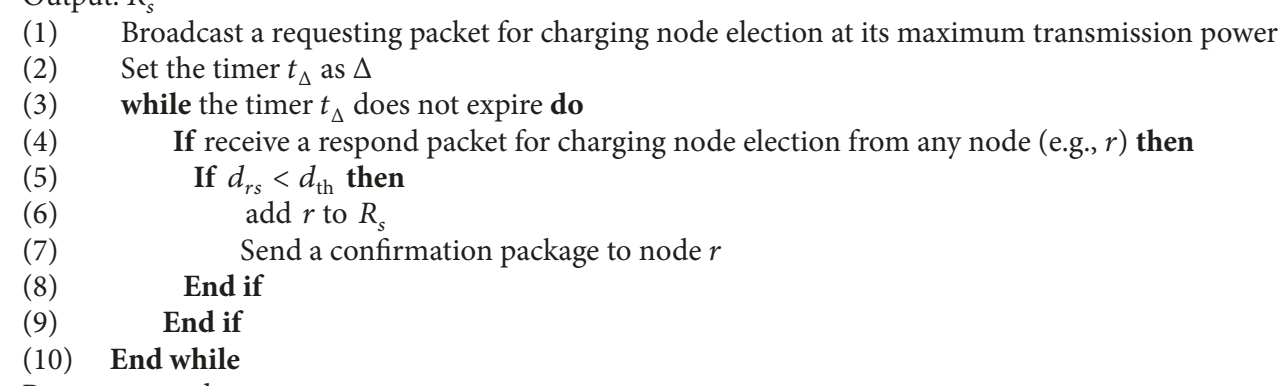

Run at any node $r$

Input: null

Output: null

(1) If receive a requesting packet for charging node election from any VAP (e.g., $s$ ) then

(2) If $d_{r s}^{2}+d_{r m}^{2}<d^{2}{ }_{s m}, 1.5<d_{r m} / d_{r s}<3$ and $e_{r}>e_{\text {th }}$ then

(3) Send a responding packet to VAP $s$

(4) End if

(5) End if

(6) If receive a confirmation package from any VAP (e.g., s) then

(7) Mark itself as the charging node of the VAP $s$

(8) End if 


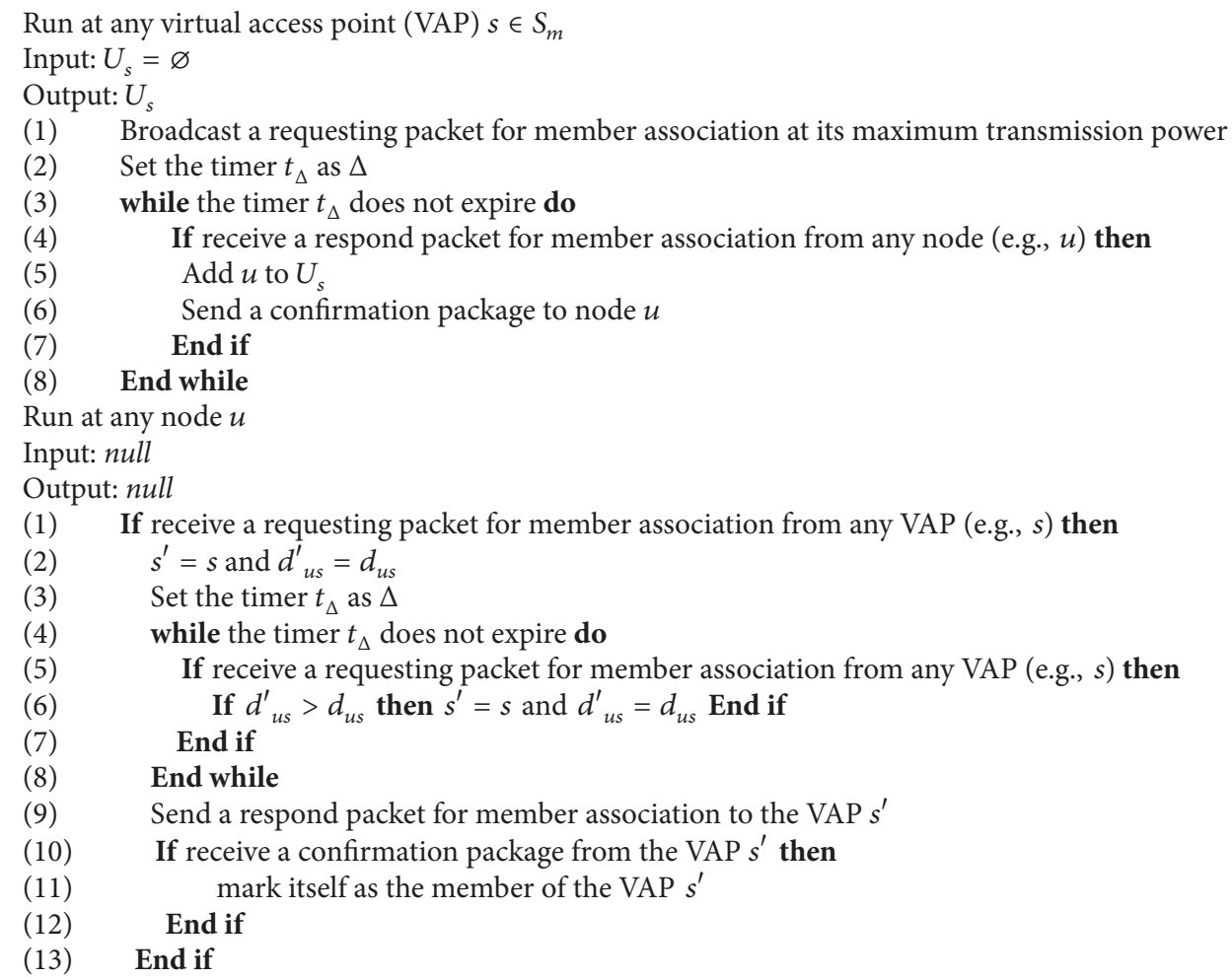

Algorithm 3: UE association for VAP.

\begin{tabular}{|c|c|c|}
\hline \multicolumn{2}{|c|}{$\beta \cdot T$} & $(1-\beta) \cdot T$ \\
\hline$\alpha \cdot \beta \cdot T$ & $(1-\alpha) \cdot \beta \cdot T$ & $(1-\beta) \cdot T$ \\
\hline Energy transfer from MBS to ETR & Energy transfer from ETR to VAP & Information transmission \\
\hline
\end{tabular}

FIGURE 2: The structure of time frame.

the downlink, while each UE piece adopts SC-FDMA and communicates with the MBS over the same number of subcarriers in the uplink. Without loss of generality, we assume that the fixed number of orthogonal subcarriers is used by the MBS to communicate with each UE piece, where the total channel frequency band for any UE is denoted as $W$. Meanwhile, it is assumed that the MBS is furnished with $\left|S_{m}\right|+$ 1 antennas at least so that it can receive the data from $\left|S_{m}\right|$ VAPs and broadcast energy to $\left|S_{m}\right| \cdot\left|R_{s}\right|$ ETRs simultaneously, while all UE pieces, including ETRs and VAPs, are equipped with one single antenna each. In this paper, $|\cdot|$ denotes the number of members in a set (e.g., $\left|S_{m}\right|$ is the number of the VAPs in $S_{m}$, while $\left|R_{s}\right|$ is the number of the ETRs in $R_{s}$ ).

The MBS divides the communication time into time frames with the same size (e.g., $T$ ). For each virtual cellular coverage area with a VAP (e.g., $s$ in $S_{m}$ ), the MBS specifies the structure of each time frame shown in Figure 2, which includes two time slots: (1) energy transfer time slot, in which ETRs harvest the energy from the MBS over frequency band $W$ during the $\alpha \cdot \beta \cdot T$ time and the VAP (i.e., $s$ ) harvests the energy from the ETRs (i.e., the members in $R_{s}$ ) over frequency band $W$ during the $(1-\alpha) \cdot \beta \cdot T$ time, with $\beta$ being the fraction of the energy transfer time in $T$ time and $\alpha$ being the fraction of the energy transfer time from the MBS to ETRs in $\beta \cdot T$ time, $0<\alpha<1$, and $0<\beta<1$; (2) information transmission time slot, in which a transmitting node sends the information signal to the corresponding receiver by using the harvested energy during the $\beta \cdot T$ time, together with its remaining battery energy.

During the $(1-\alpha) \cdot \beta \cdot T$ time of each time frame, the VAP (i.e., $s$ ) receives energy from its $\left|R_{s}\right|$ ETRs in TDMA mode. In other words, each ETR will only transmit its energy during its time slot with length $\tau_{r} \cdot(1-\alpha) \cdot \beta \cdot T$, where $0<\tau_{r}<1$, $\forall r \in\left\{1,2, \ldots,\left|R_{s}\right|\right\}$, and $\sum_{r \in\left\{1,2, \ldots, R_{s} \mid\right\}} \tau_{r}=1$. Also, during the $(1-\beta) \cdot T$ time of each time frame, the VAP (i.e., $s$ ) receives the data from its $\left|U_{s}\right|$ associated UE pieces in TDMA mode. In other words, each UE piece will only transmit its data during its time slot with length $\varepsilon_{u} \cdot(1-\beta) \cdot T$, where $0<\varepsilon_{u}<1, \forall u \in$ $\left\{1,2, \ldots,\left|U_{s}\right|\right\}$, and $\sum_{u \in\left\{1,2, \ldots,\left|U_{s}\right|\right\}} \varepsilon_{u}=1$. The timing diagram is shown Figure 3. 


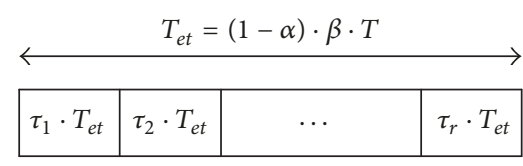

(a) Energy transfer from ETR to VAP

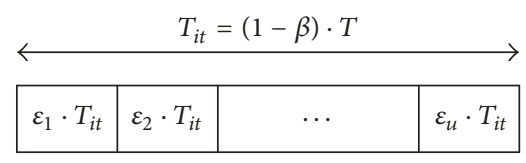

(b) Information transmission from UE to VAP

FIgURE 3: The timing diagram.

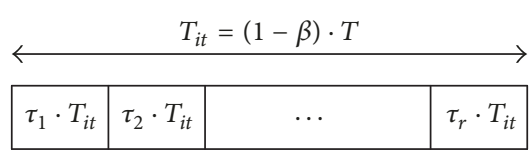

(a) Information transmission from ETR to MBS

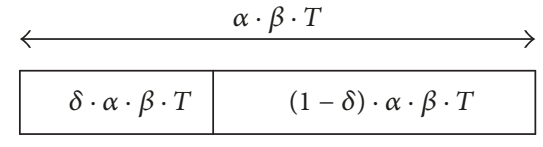

(b) Information transmission from VAP to MBS

FIgURE 4: The time slot multiplexing.

Although an ETR (e.g., $r$ ) receives energy from the MBS during the $\alpha \cdot \beta \cdot T$ time of a time frame and sends energy to the VAP during the $(1-\alpha) \cdot \beta \cdot T$ time of the same time frame, respectively, it can send its data to the MBS in the part of the $(1-\beta) \cdot T$ time of the same time frame, that is, $\tau_{r} \cdot(1-\beta) \cdot T$ time, as shown in Figure $4(\mathrm{a})$. Here, since the ETR reuses UE's transmitting time slot, it should employ the different frequency bands.

Also, for the data received by the VAP in the $(1-\beta) \cdot T$ time of the previous time frame, the VAP can send them to the MBS in the part of the $\alpha \cdot \beta \cdot T$ time of the current time frame, that is, $\delta \cdot \alpha \cdot \beta \cdot T$ time and $0<\delta<1$, while $(1-\delta)$. $\alpha \cdot \beta \cdot T$ time is used by the VAP to send its own data to the MBS, as shown in Figure 4(b). Here, since the VAP reuses the MBS's transmitting time slot, it should employ the different frequency bands.

3.3. Problem Formulation and Theoretical Analysis. In this subsection, we first describe wireless energy transfer model and wireless information transmission model and then develop a game-theoretic framework for virtual cellular coverage area construction.

After the MBS (as a leader) offers an initial charging power to all the ETRs, each ETR (as a follower) determines its charging power to its associating VAP. Once each VAP (as a follower) receives the charging powers from all the ETRs associated with it, it determines its transmission power for relaying data from its associating UE pieces to the MBS. Since an ETR charging power is subject to the MBS, it is more appropriate that the MBS acts as the leader for the VAPs.

We will analyze the proposed Stackelberg game based on three-party circular decision and obtain its SNE of this game. Based on our analysis, we know that each party's strategy affects other parties' strategies. Therefore, a reverse induction method can be used to analyze the proposed game, because it can capture the sequential dependence of the decisions in all the game stages.
3.3.1. Wireless Signal Propagation Model. When a receiver $j$ works in the energy harvesting mode, the power harvested from source $i$ can be calculated as follows:

$$
p_{i, j}^{r}=\eta_{i, j} \cdot p_{i, j}^{t} \cdot\left|h_{i, j}\right|^{2}
$$

In (1), $\eta_{i, j}$ denotes the power conversion efficiency factor from node $i$ to node $j, p_{i, j}^{t}$ is the transmission power at source $i, p_{i, j}^{r}$ is the harvested power at receiver $j$, and $h_{i, j}$ denotes the channel power gain between source $i$ and receiver $j$. Here, let $g_{i, j}=\left|h_{i, j}\right|^{2}$, which is calculated by using the following formula $[22,36]$ :

$$
g_{i, j}=10^{-\left(\mathrm{PL}_{\mathrm{ref}}+\psi_{i, j}\right) / 10} \cdot d_{i, j}^{-\varphi} \cdot \beta_{i, j}
$$

In (2), $\varphi$ is path loss exponent; $\mathrm{PL}_{\text {ref }}$ is the path loss in $\mathrm{dB}$ at the reference distance; $\psi_{i, j}$ is the body shadowing loss margin between source $i$ and receiver $j$ in $\mathrm{dB}, d_{i, j}$ is the distance between source $i$ and receiver $j ; \beta_{i, j}$ is the smallscale fading power gain between source $i$ and receiver $j$. For simplicity, the paper assumes $\eta_{i, j}=\eta$ and $\psi_{i, j}=\psi$.

Let $W$ and $\sigma^{2}$ denote the transmission bandwidth and noise power, respectively. When the receiver $j$ works in the information decoding mode, the information decoding rate $b_{i, j}$ from source $i$ to receiver $j$ is as follows:

$$
b_{i, j}=W \cdot \log _{2}\left(1+\frac{p_{i, j}^{t} \cdot g_{i, j}}{\sigma^{2}}\right) \text {. }
$$

3.3.2. Game Formulation and Theoretical Analysis for ETRs. In the subsection, we consider a noncooperative scenario in which the ETRs are all rational and self-interested such that they only want to minimize their charging powers and maximize their transmitting powers. Therefore, we formulate the noncooperative game for the ETRs, where the game players are the $\left|R_{s}\right|$ ETRs, the game actions are that each 
ETR determines the charging power and transmitting power under the constraint of its maximum transmitting power, and the game utility is the data rate for each ETR.

For any ETR (e.g., $r$ ), when the MBS (i.e., $m$ ) broadcasts energy at $p_{m, r}^{t}$, its harvested power $p_{m, r}^{r}$ can be calculated according to formula (1) and specified as $p_{m, r}^{r}=\eta \cdot p_{m, r}^{t} \cdot g_{m, r}$. Also, when the maximum transmission power of the ETR $r$ is set as $p_{r}^{\max }$, the power used in transferring energy from $r$ to $s \in S_{m}$ (i.e., $p_{r, s}^{t}$ ) and that used in transmitting data from $r$ to $m$ (i.e., $p_{r, m}^{t}$ ) have to meet the following relation.

$$
p_{r}^{\max } \geq p_{r, s}^{t}+p_{r, m}^{t}
$$

On the one hand, the ETR $r$ should employ the more value of $p_{r, s}^{t}$ to transfer energy to the VAP in order to obtain the more free traffic access to Internet; on the other hand, it has to use more value of $p_{r, m}^{t}$ to transmit its own data such that the free traffic share is fully utilized. Therefore, the ETR $r$ must determine optimal transmission power splitting ratio under the constraint of $p_{r}^{\max }$.

When the ETR $r$ adopts $p_{r}^{\max }$ and its battery energy storage and harvested power are $E_{r}$ and $p_{m, r}^{r}$, respectively, its continuous emission time (i.e., $T_{r}^{\max }$ ) can be estimated by the following formula:

$$
T_{r}^{\max }=\frac{E_{r}}{p_{r}^{\max }-p_{m, r}^{r}} .
$$
follows:

During $T_{r}^{\max }$, the utility of the ETR $r$ is represented as

$$
\begin{aligned}
& \mu_{r}\left(P_{r}\right) \\
& \quad=T_{r}^{\mathrm{max}} \cdot \varphi_{r, m} \cdot W_{r} \cdot \log _{2}\left(1+\frac{\left(p_{r}^{\mathrm{max}}-p_{r, s}^{t}\right) \cdot g_{r, m}}{\sigma^{2}}\right) \\
& \varphi_{r, m}=\frac{\log _{2}\left(1+\left(\eta \cdot p_{r, s}^{t} \cdot g_{r, s} \cdot g_{s, m}\right) / \sigma^{2}\right)}{\emptyset_{r}+\sum_{r^{\prime} \in R_{s}} \log _{2}\left(1+\left(\eta \cdot p_{r^{\prime}, s}^{t} \cdot g_{r^{\prime}, s} \cdot g_{s, m}\right) / \sigma^{2}\right)} \\
& P_{r}=\left\{p_{r^{\prime}, s}^{t} \mid \forall r^{\prime} \in R_{s}\right\} .
\end{aligned}
$$

In (6), $\emptyset_{r}$ is seen as the contribution from a fictitious selfless node and usually set to a constant (e.g., $10^{-7}$ ), where the aim is to prevent game participants from colluding to cheat. We notice that each player's strategy (e.g., $p_{r, s}^{t}$ of the ETR $r$ ) only depends on the strategies of the other players (i.e., $\left.P_{-r}=\left\{p_{r^{\prime}, s}^{t} \mid \forall r^{\prime} \in R_{s} \backslash p_{r, s}^{t}\right\}\right)$. Thus, given the strategies of the other players $P_{-r}$, the best response strategy of the ETR $r$ is the solution to the following optimization problem. That is, the ETR $r$ needs to find the optimal transmission power $p_{r, s}^{t}$ to offer to the VAP $s$ to maximize its individual profit, which can be obtained by solving the following optimization problem:

$$
\begin{aligned}
& \max _{\left\{p_{r, s}^{t}, P_{-r}\right\}}\left\{T_{r}^{\max } \cdot \varphi_{r, m} \cdot W_{r}\right. \\
& \left.\cdot \log _{2}\left(1+\frac{\left(p_{r}^{\max }-p_{r, s}^{t}\right) \cdot g_{r, m}}{\sigma^{2}}\right)\right\} .
\end{aligned}
$$

We denote by $p_{r, s}^{t *}$ the optimal solution to optimization problem (7). It is straightforward to verify that (7) always has a feasible solution. A SNE of the formulated game is a feasible profile $p_{r, s}^{t *}$ that satisfies (7). Now, we calculate the best response strategy $p_{r, s}^{t *}$ for the ETR $r$ by solving optimization problem (7), which is described in the following theorem.

Theorem 1. Given $P_{-r}=\left\{p_{r^{\prime}, s}^{t} \mid \forall r^{\prime} \in R_{s} \backslash\left\{p_{r, s}^{t}\right\}\right.$, the optimal response strategy $p_{r, s}^{t *}$ of the ETR $r$ can be expressed as follows:

$$
p_{r, s}^{t *}=\frac{A}{B}
$$

where

$$
\begin{aligned}
A= & \eta \cdot g_{r, s} \cdot g_{s, m} \cdot \sigma^{2} \cdot\left(1-\varphi_{r, m}\right) \cdot X_{r}+\eta \cdot g_{r, s} \cdot g_{s, m} \\
& \cdot g_{r, m} \cdot\left(1-\varphi_{r, m}\right) \cdot p_{r}^{\max } \cdot X_{r}-g_{r, m} \cdot \sigma^{2} \cdot \varphi_{r, m} \\
& \cdot Y_{r} \\
B= & \eta \cdot g_{r, s} \cdot g_{s, m} \cdot g_{r, m} \cdot \varphi_{r, m} \cdot Y_{r}+\eta \cdot g_{r, s} \cdot g_{s, m} \cdot g_{r, m} \\
& \cdot\left(1-\varphi_{r, m}\right) \cdot X_{r} \\
X_{r}= & \log _{2}\left(1+\frac{\left(p_{r}^{\max }-p_{r, s}^{t}\right) \cdot g_{r, m}}{\sigma^{2}}\right) \\
Y_{r}= & \sum_{r^{\prime} \in R_{s}} \log _{2}\left(1+\frac{\eta \cdot p_{r^{\prime}, s}^{t} \cdot g_{r^{\prime}, s} \cdot g_{s, m}}{\sigma^{2}}\right) .
\end{aligned}
$$

Proof. To maximize its net utility, each ETR hopes to determine an optimal transmission power splitting ratio. For an arbitrary ETR $r$, its net utility function defined in (6) is a concave function of $p_{r, s}^{t}$ since $\partial^{2} \mu_{r}\left(P_{r}\right) / \partial\left(p_{r, s}^{t}\right)^{2}<0$. Therefore, let $\partial \mu_{r}\left(P_{r}\right) / \partial p_{r, s}^{t}=0$; the optimal response strategy $p_{r, s}^{t *}$ of the ETR $r$ can be denoted as (8). This completes the proof.

In fact, this power $p_{r, s}^{t *}$ cannot be solved directly from formula (8) since it is included in $A$ and $B$ of formula (8). However, due to the concave characteristic stated in Theorem 1, a new approximating algorithm (i.e., Algorithms 4) is proposed in the following text to obtain its approximate optimal value.

3.3.3. Game Formulation and Theoretical Analysis for VAPs. In the subsection, we consider a noncooperative scenario in which the VAPs are all rational and self-interested such that they only want to minimize their forwarding powers and maximize their transmitting powers. Therefore, we formulate the noncooperative game for the VAPs, where the game players are $\left|S_{m}\right|$ VAPs, the game actions are that each VAP determines the forwarding power and transmitting power under the constraint of its maximum transmitting power, and the game utility is the data rate for each VAP. For any $\operatorname{VAP}\left(\right.$ e.g., $s$ ), its harvesting energy from the $\left|R_{s}\right|$ ETRs can be estimated by the following formula:

$$
e_{s}=\sum_{r \in R_{s}} T_{r}^{\max } \cdot \eta \cdot p_{r, s}^{t} \cdot g_{r, s} \cdot
$$


Also, when the maximum transmission power of the VAP $s$ is set as $p_{s}^{\max }$, the power used in forwarding data from UE to the MBS $m$ (i.e., $p_{s, m}^{t}$ ) and that used in transmitting its own data to the MBS $m$ (i.e., $p_{u, m}^{t}$ ) have to meet the following relation:

$$
p_{s}^{\max } \geq p_{s, m}^{t}+p_{u, m}^{t}
$$

On the one hand, the VAP $s$ should employ more value of $p_{s, m}^{t}$ in forwarding data to the MBS $m$ in order to obtain the more free traffic access to Internet; on the other hand, it has to use more value of $p_{u, m}^{t}$ to transmit its own data such that the free traffic share is fully utilized.

Therefore, the VAP $s$ must determine optimal transmission power splitting ratio under the constraint of $p_{s}^{\max }$. When the VAP $s$ adopts $p_{s}^{\max }$ and its battery energy storage and harvested energy from the ETRs are $E_{s}$ and $e_{s}$, respectively, its continuous emission time (i.e., $T_{s}^{\max }$ ) can be estimated by the following formula:

$$
T_{s}^{\max }=\frac{E_{s}+e_{s}}{p_{s}^{\max }}
$$
follows:

During $T_{s}^{\max }$, the utility of the VAP $s$ is represented as

$$
\begin{aligned}
\mu_{s}\left(P_{s}\right)= & T_{s}^{\max } \cdot \varphi_{s, m} \cdot W_{s} \\
& \cdot \log _{2}\left(1+\frac{\left(p_{s}^{\max }-p_{s, m}^{t}\right) \cdot g_{s, m}}{\sigma^{2}}\right) \\
\varphi_{s, m}= & \frac{\log _{2}\left(1+\left(p_{s, m}^{t} \cdot g_{s, m}\right) / \sigma^{2}\right)}{\emptyset_{s}+\sum_{s^{\prime} \in S_{m}} \log _{2}\left(1+\left(p_{s^{\prime}, m}^{t} \cdot g_{s^{\prime}, m}\right) / \sigma^{2}\right)} \\
P_{s}= & \left\{p_{s^{\prime}, m}^{t} \mid \forall s^{\prime} \in S_{m}\right\} .
\end{aligned}
$$

In (13), the meaning of $\emptyset_{s}$ is similar to $\emptyset_{r}$ and also set to a constant (e.g., 3). We notice that each player's strategy (e.g., $p_{s, m}^{t}$ of the VAP $s$ ) only depends on the strategies of the other players (i.e., $P_{-s}=\left\{p_{s^{\prime}, m}^{t} \mid \forall s^{\prime} \in S_{m} \backslash\left\{p_{s, m}^{t}\right\}\right\}$ ). Thus, given the strategies of the other players $P_{-s}$, the best response strategy of the VAP $s$ is the solution to the following optimization problem. That is, the node $s$ needs to find the optimal forwarding power $p_{s, m}^{t}$ to offer data forwarding service for its associating UE pieces in order to maximize its individual profit, which can be obtained by solving the following optimization problem:

$$
\begin{aligned}
& \max _{\left\{p_{s, m}^{t}, P_{-s}\right\}}\left\{T_{s}^{\max } \cdot \varphi_{s, m} \cdot W_{s}\right. \\
& \left.\cdot \log _{2}\left(1+\frac{\left(p_{s}^{\max }-p_{s, m}^{t}\right) \cdot g_{s, m}}{\sigma^{2}}\right)\right\} .
\end{aligned}
$$

We denote by $p_{s, m}^{t *}$ the optimal solution to optimization problem (14). It is straightforward to verify that (14) always has a feasible solution. A SNE of the formulated game is a feasible profile $p_{s, m}^{t *}$ that satisfies (14). Now, we calculate the best response strategy $p_{s, m}^{t *}$ for the VAP $s$ by solving optimization problem (14), which is described in the following theorem.
Theorem 2. Given $P_{-s}=\left\{p_{s^{\prime}, m}^{t} \mid \forall s^{\prime} \in S_{m} \backslash\left\{p_{s, m}^{t}\right\}\right\}$, the optimal response strategy $p_{s, m}^{t *}$ of the VAP $s$ can be expressed as follows:

$$
p_{s, m}^{t *}=\frac{C}{D}
$$

where

$$
\begin{aligned}
C= & g_{s, m} \cdot \sigma^{2} \cdot\left(1-\varphi_{s, m}\right) \cdot X_{s}+g_{s, m}^{2} \cdot\left(1-\varphi_{s, m}\right) \\
& \cdot p_{s}^{m a x} \cdot X_{s}-g_{s, m} \cdot \sigma^{2} \cdot \varphi_{s, m} \cdot Y_{s} \\
D= & g_{s, m}^{2} \cdot \varphi_{s, m} \cdot Y_{s}+g_{s, m}^{2} \cdot\left(1-\varphi_{s, m}\right) \cdot X_{s} \\
X_{s}= & \log _{2}\left(1+\frac{\left(p_{s}^{m a x}-p_{s, m}^{t}\right) \cdot g_{s, m}}{\sigma^{2}}\right) \\
Y_{s}= & \sum_{s^{\prime} \in S_{m}} \log _{2}\left(1+\frac{p_{s^{\prime}, m}^{t} \cdot g_{s^{\prime}, m}}{\sigma^{2}}\right) .
\end{aligned}
$$

Proof. To maximize its net utility, each VAP hopes to determine an optimal transmission power splitting ratio. For an arbitrary VAP $s$, its net utility function defined in (13) is a concave function of $p_{s, m}^{t}$ since $\partial^{2} \mu_{s}\left(P_{s}\right) / \partial\left(p_{s, m}^{t}\right)^{2}<0$. Therefore, let $\partial \mu_{s}\left(P_{s}\right) / \partial p_{s, m}^{t}=0$, the optimal response strategy $p_{s, m}^{t *}$ of the VAP $s$ can be denoted as (15). This completes the proof.

Similar to the discussion and solution for $p_{r, s}^{t *}$, we also provide a new approximate solution (i.e., Algorithms 5) in the following text to solve $p_{s, m}^{t *}$.

3.3.4. Game Formulation and Theoretical Analysis for MBS. In the subsection, we mainly focus on how the decision in the MBS is affected by the game results of ETRs and VAPs. Based on the conditions described in the previous text, the Stackelberg game theory, including leaders and followers competing with each other for certain resources, applies to this scenario.

In this paper, the MBS acts as the leader and offers an initial charging power to the ETRs with its maximum transmission power. The purpose is to encourage all UE pieces to participate actively. Following this, the ETRs and their associating VAP act as the followers and make their decisions according to their utility functions, respectively. According to the feedback from the followers, the leader may readjust its strategy.

It is assumed that the MBS broadcasts energy to $\left|S_{m}\right|$. $\left|R_{s}\right|$ ETRs with omnidirectional antenna. Therefore, for an arbitrary ETR (e.g., $r$ ), the charging power $p_{m, r}^{t}$ is simplified as $p_{m}^{t}$. The MBS hopes to obtain the maximum forwarding traffic from the $\left|S_{m}\right|$ VAPs but just wants to pay the minimum price of energy. Therefore, the utility of the MBS $m$ is represented as follows:

$$
\begin{aligned}
\mu_{m}\left(p_{m}^{t}, P_{r}, P_{s}\right)= & c_{b} \cdot \sum_{s \in S_{m}} T_{s}^{\max } \cdot W \\
& \cdot \log _{2}\left(1+\frac{p_{s, m}^{t} \cdot g_{s, m}}{\sigma^{2}}\right)-c_{e} \\
& \cdot \max \left\{T_{s}^{\max } \mid s \in S_{m}\right\} \cdot p_{m}^{t} .
\end{aligned}
$$


In (17), $c_{b}$ is traffic price per bit, while $c_{e}$ is energy price per joule. According to formulas (1), (5), (10), and (12), $T_{s}^{\max }$ can be rewritten as follows:

$$
T_{s}^{\max }=f_{s}^{T}\left(p_{m}^{t}, P_{r}, P_{s}\right)=\frac{E_{s}+\sum_{r \in R_{s}}\left(\left(E_{r} \cdot \eta \cdot\left(p_{r}^{\max }-p_{r, m}^{t}\right) \cdot g_{r, s}\right) /\left(p_{r}^{\max }-\eta \cdot p_{m}^{t} \cdot g_{m, r}\right)\right)}{p_{s}^{\max }} .
$$

Thus, given $P_{r}$ and $P_{s}$ from the ETRs and VAPs, respectively, the best response strategy of the MBS $m$ is the solution to the following optimization problem:

$$
\begin{aligned}
& \max _{\left\{p_{m}^{t}, P_{r}, P_{s}\right\}}\left\{c_{b} \cdot \sum_{s \in S_{m}} T_{s}^{\max } \cdot W \cdot \log _{2}\left(1+\frac{p_{s, m}^{t} \cdot g_{s, m}}{\sigma^{2}}\right)\right. \\
& \left.-c_{e} \cdot \max \left\{T_{s}^{\max } \mid s \in S_{m}\right\} \cdot p_{m}^{t}\right\} .
\end{aligned}
$$

Now, we calculate the best response strategy $p_{m}^{t *}$ for the MBS $m$ by solving optimization problem (19), which is described in the following theorem.

Theorem 3. Given $P_{r}$ and $P_{s}$ from the ETRs and VAPs, respectively, when the following relation is met,

$$
p_{m}^{t}>\left[\frac{c_{b} \cdot \sum_{s \in S_{m}}\left(\partial^{2} T_{s}^{\max } / \partial\left(p_{m}^{t}\right)^{2}\right) \cdot W \cdot \log _{2}\left(1+\left(p_{s, m}^{t} \cdot g_{s, m}\right) / \sigma^{2}\right)-2 c_{e} \cdot \max \left\{\partial T_{s}^{\max } / \partial p_{m}^{t} \mid s \in S_{m}\right\}}{c_{e} \cdot \max \left\{\partial^{2} T_{s}^{\max } / \partial\left(p_{m}^{t}\right)^{2} \mid s \in S_{m}\right\}}\right]^{+},
$$

the optimal response strategy $p_{m}^{t *}$ of the $M B S m$ can be expressed as follows:

$$
p_{m}^{t *}=\left[\frac{c_{b} \cdot \sum_{s \in S_{m}}\left(\partial T_{s}^{\max } / \partial p_{m}^{t}\right) \cdot W \cdot \log _{2}\left(1+\left(p_{s, m}^{t} \cdot g_{s, m}\right) / \sigma^{2}\right)-c_{e} \cdot \max \left\{T_{s}^{\max } \mid s \in S_{m}\right\}}{c_{e} \cdot \max \left\{\partial T_{s}^{\max } / \partial p_{m}^{t} \mid s \in S_{m}\right\}}\right]^{+},
$$

where

$$
\begin{aligned}
& {[\cdot]^{+} \triangleq \max \{\cdot, 0\}} \\
& \frac{\partial T_{s}^{\max }}{\partial p_{m}^{t}} \\
& \quad=\frac{1}{p_{s}^{\max }} \cdot \sum_{r \in R_{s}} \frac{E_{r} \cdot \eta^{2} \cdot g_{m, r} \cdot g_{r, s} \cdot\left(p_{r}^{\max }-p_{r, m}^{t}\right)}{\left(p_{r}^{\max }-\eta \cdot p_{m}^{t} \cdot g_{m, r}\right)^{2}} \\
& \frac{\partial^{2} T_{s}^{\max }}{\partial\left(p_{m}^{t}\right)^{2}} \\
& \quad=\frac{1}{p_{s}^{\max }} \cdot \sum_{r \in R_{s}} \frac{2 E_{r} \cdot \eta^{3} \cdot g_{m, r}^{2} \cdot g_{r, s} \cdot\left(p_{r}^{\max }-p_{r, m}^{t}\right)}{\left(p_{r}^{\max }-\eta \cdot p_{m}^{t} \cdot g_{m, r}\right)^{3}} .
\end{aligned}
$$

Proof. The optimization problem with respect to (19) always has a feasible solution under the condition that the first-order derivative of $\mu_{m}\left(p_{m}^{t}, P_{r}, P_{s}\right)$ with respect to $p_{m}^{t}$, that is, $\partial^{2} \mu_{m}\left(p_{m}^{t}, P_{r}, P_{s}\right) / \partial\left(p_{s, m}^{t}\right)^{2}$, is less than 0 . Since $\partial^{2} \mu_{m}\left(p_{m}^{t}, P_{r}, P_{s}\right) / \partial\left(p_{s, m}^{t}\right)^{2}$ can be denoted as $c_{b} \cdot \sum_{s \in S_{m}}\left(\partial^{2} T_{s}^{\max } /\right.$ $\left.\partial\left(p_{m}^{t}\right)^{2}\right) \cdot W \cdot \log _{2}\left(1+\left(p_{s, m}^{t} \cdot g_{s, m}\right) / \sigma^{2}\right)-c_{e} \cdot \max \left\{\partial^{2} T_{s}^{\max } / \partial\left(p_{m}^{t}\right)^{2} \mid\right.$ $\left.s \in S_{m}\right\} \cdot p_{m}^{t}-2 c_{e} \cdot \max \left\{\partial T_{s}^{\max } / \partial p_{m}^{t} \mid s \in S_{m}\right\}$, and it will be less than 0 according to conditions (23) and (24) when expression (20) is met.

By solving the first-order derivative of $\mu_{m}\left(p_{m}^{t}, P_{r}, P_{s}\right)$ with respect to $p_{m}^{t}$, we can obtain the following expression:

$$
\begin{aligned}
\frac{\partial \mu_{m}\left(p_{m}^{t}, P_{r}, P_{s}\right)}{\partial p_{m}^{t}}= & c_{b} \cdot \sum_{s \in S_{m}} \frac{\partial^{2} T_{s}^{\max }}{\partial\left(p_{m}^{t}\right)^{2}} \cdot W \\
& \cdot \log _{2}\left(1+\frac{p_{s, m}^{t} \cdot g_{s, m}}{\sigma^{2}}\right)-c_{e} \\
& \cdot \max \left\{\frac{\partial^{2} T_{s}^{\max }}{\partial\left(p_{m}^{t}\right)^{2}} \mid s \in S_{m}\right\} \cdot p_{m}^{t} \\
& -2 c_{e} \cdot \max \left\{\frac{\partial T_{s}^{\max }}{\partial p_{m}^{t}} \mid s \in S_{m}\right\} .
\end{aligned}
$$


TABLE 1: The game scenarios in our work.

\begin{tabular}{llcl}
\hline Scenario & Actor & Strategy & Payoff \\
\hline ETR game & ETRs & $\begin{array}{c}\text { Each player hopes to find the optimal } \\
\text { charging power offered to its associating } \\
\text { VAP to maximize its individual profit. }\end{array}$ & $\begin{array}{c}\text { The utility of any player is computed by } \\
\text { formula (6). }\end{array}$ \\
\hline VAP game & VAPs & $\begin{array}{c}\text { Each player hopes to find the optimal } \\
\text { forwarding power to offer data } \\
\text { forwarding service for its associating UEs } \\
\text { to maximize its individual profit. }\end{array}$ & $\begin{array}{c}\text { The utility of any player is computed by } \\
\text { formula (13). }\end{array}$ \\
$\begin{array}{l}\text { Stackelberg } \\
\text { game }\end{array}$ & $\begin{array}{c}\text { The MBS hopes to obtain the maximum } \\
\text { ETRs }\end{array}$ & $\begin{array}{c}\text { The utility of the MBS is computed by } \\
\text { formula (17). } \\
\text { VAPs }\end{array}$ & $\begin{array}{c}\text { wants to pay the minimum price of } \\
\text { energy. }\end{array}$ \\
\hline
\end{tabular}

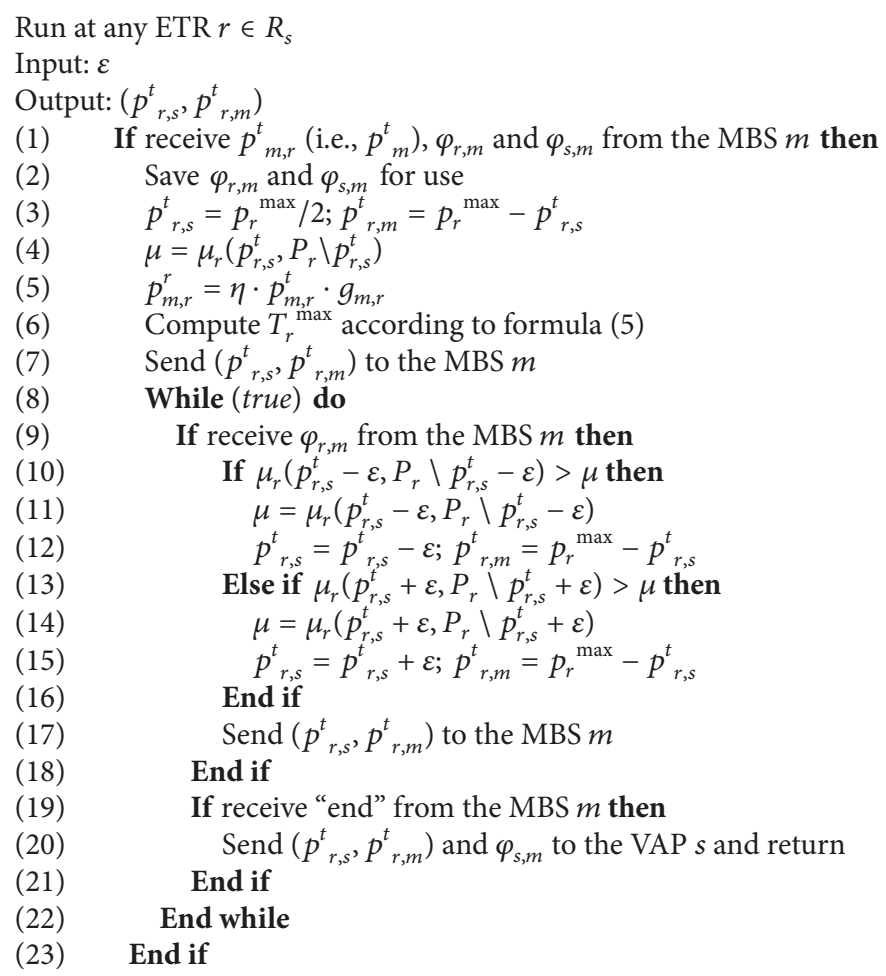

Algorithm 4: ETR-level game.

Let expression (25) (i.e., the first-order derivative of $\mu_{m}\left(p_{m}^{t}, P_{r}, P_{s}\right)$ with respect to $\left.p_{m}^{t}\right)$ be equal to 0 ; we can obtain expression (21) with some algebra manipulations, which completes the proof.

From Theorem 3, we know that expression (19) satisfies the concavity under a given condition. Therefore, if $p_{m}^{t}$ that satisfies expression (20) is less than MBS maximum power $p_{m}^{\max }$, we can design an approximate algorithm to search the approximate value for $p_{m}^{t *}$ in the interval from $\left[p_{m}^{t}\right]^{+}$to $p_{m}^{\max }$.

To facilitate readers' understanding, Table 1 summarizes the game scenarios, actors (or players), strategies (or actions), and payoffs (or utilities) in this paper.
3.4. Distributed Iterative Algorithms for Obtaining SNE. Since we have derived the optimal transmission power splitting levels for ETRs and VAPs to achieve their desired data rates (i.e., from their own to the MBS), as well as allowing the MBS to achieve its maximum utility by adjusting its charging power, we now propose the three efficient and practical algorithms that find the approximately optimal power levels, which are based on the results of theoretical analysis in Section 3.3.

Based on a reverse induction mode, the system interaction is as follows. Given an initial charging power offered by the MBS to the ETRs, (1) each ETR firstly determines its charging power to its associating VAP by running Algorithm 4; (2) then each VAP determines its transmission 


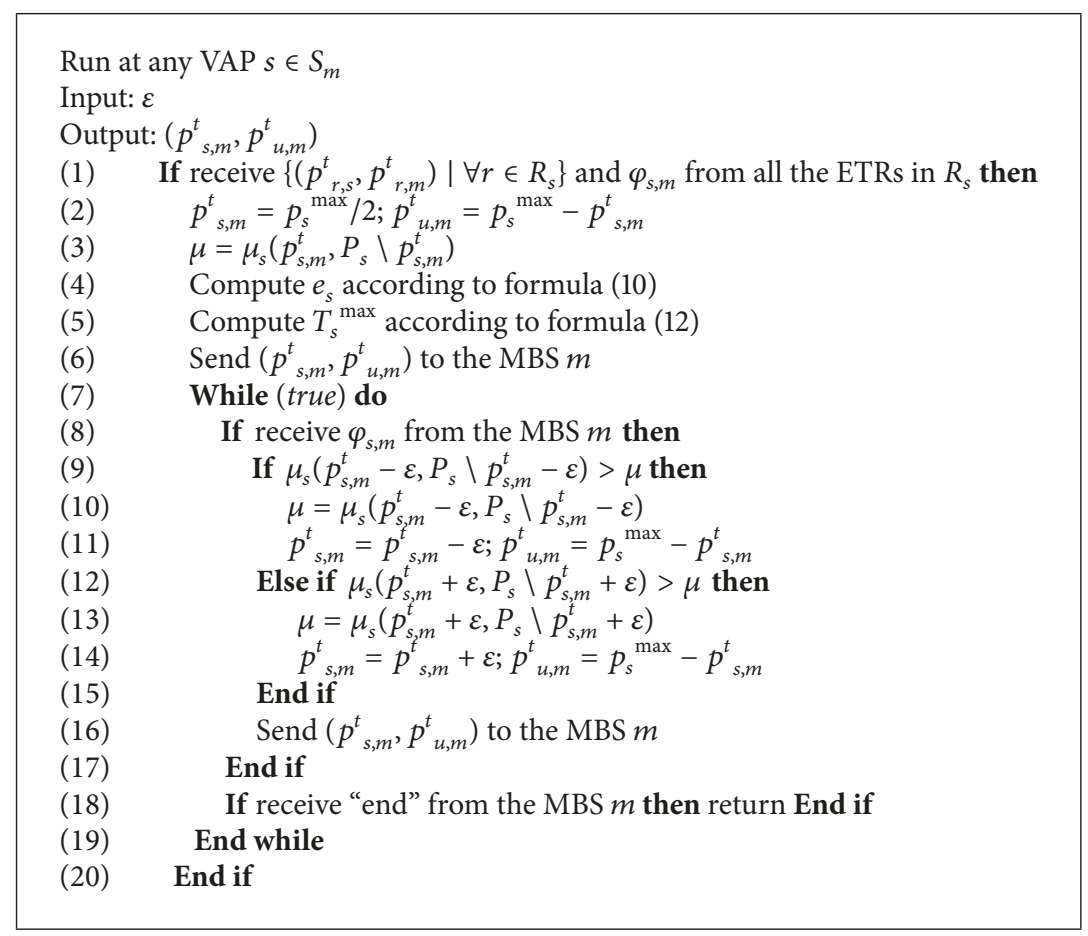

Algorithm 5: VAP-level game.

for relaying data from its associating UE pieces to the MBS according to the results of Algorithm 5; (3) finally, the MBS updates its charging power to the ETRs by executing Algorithm 6. The steps (1), (2), and (3) are repeated until the powers converge.

Parameter $\varepsilon$ is set to a small value (e.g., $0.1 \% \sim 3.5 \%$ ). In any of the three algorithms, the better response of any player in the current round is the better transmission power splitting levels or charging power offered to maximize its total revenue, given those offered by the other players at the previous round of the iteration process.

\section{Performance Evaluation}

4.1. Simulation Setting. In our simulations, the macrocell network consists of one MBS and a set of UE pieces (denoted as $\varnothing)$, where the number of members in $\varnothing$ is denoted as $|\varnothing|$. We assume that $|\varnothing|$ is 1000 . For any UE $i$, the initial battery capacity $E_{i}$ is randomly distributed from $0.05 X$ to $0.2 X$ Joule, where $X$ can take different values ranging from $20 \%$ to $80 \%$. Therefore, the average initial battery capacity $E_{\mathrm{av}}$ is obtained by the following formula.

$$
E_{\mathrm{av}}=\frac{\sum_{i \in \varnothing} E_{i}}{|\varnothing|}
$$

We assume that the coverage radius of the MBS is $500 \mathrm{~m}$, and the UE pieces are randomly located in the macrocell. Each ETR or VAP elected from UE pieces has more battery energy reserve, which can improve the network performance. The UE pieces associated with each VAP are basically located near the edge of the macrocell, since such UE pieces need the

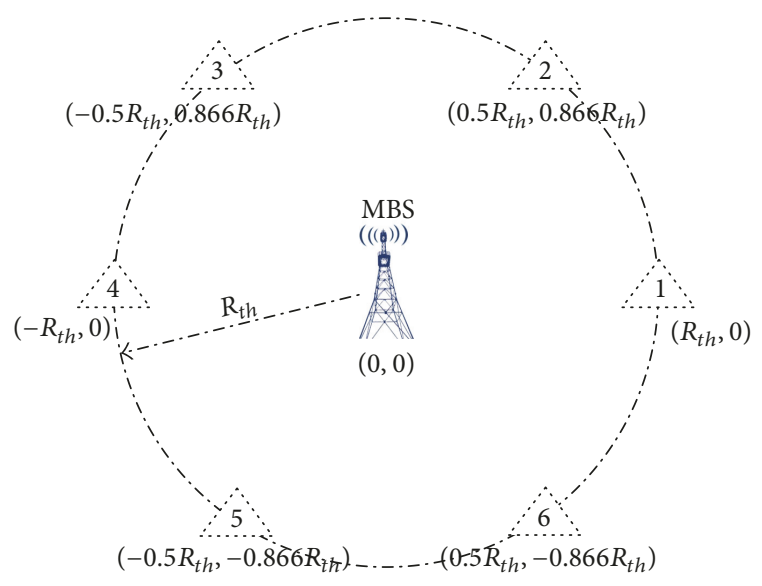

FIGURE 5: The coordinates of reference points.

help of VAPs more. We set the coordinates of reference points as shown in Figure 5, where $R_{t h}$ is valued at $100 \mathrm{~m}$.

It is assumed that channel noise power $\sigma^{2}$ (at the receivers of all UE pieces, including ETRs and VAPs) is valued at a fixed value, which is randomly distributed from -120 to $-60 \mathrm{dBm} / \mathrm{MHz}$. The other simulation parameters are listed in Table 2 .

4.2. Simulation Schemes and Metrics. In this subsection, we evaluate the performance of the scheme in this paper via simulations under a few typical values with respect to the time 
Run at the MBS $m$

Input: $\varepsilon$

Output: $p_{m}^{t}$

(1) Initialize $p_{m}^{t}$ and $\mu$ as $p_{m}{ }^{\max }$ and 0 respectively

(2) Initialize count_r and count_s as 0 respectively

(3) For each ETR $r$ do $\left\{p_{r, s}^{t}=0 ; p_{r, m}^{t}=0\right.$; count_ $\left.r++\right\}$ End for

(4) For each VAP $s$ do $\left\{p_{s, m}^{t}=0 ; p_{u, m}^{t}=0\right.$; count $\left.s++\right\}$ End for

(5) Initialize $\varphi_{r, m}$ as count_s/count_r

(6) Initialize $\varphi_{s, m}$ as $1 /$ count $\_s$

(7) Broadcast $p_{m}^{t}, \varphi_{r, m}$ and $\varphi_{s, m}$ to all the ETRs

(8) While (true)

(9) If receive the updated $\left(p_{r, s}^{t}, p_{r, m}^{t}\right)$ from any ETR $r$ then

(10) Replace the old value of $\left(p_{r, s}^{t}, p_{r, m}^{t}\right)$ with its new value

(11) Else if receive the old $\left(p_{r, s}^{t}, p_{r, m}^{t}\right)$ from any ETR $r$ then

(12) Record the cumulative number of ETRs sending old values

(13) End if

(14) If all the ETRs report $\left(p_{r, s}^{t}, p_{r, m}^{t}\right)$ and there is a new value then

(15) For each ETR $r$ do compute $\varphi_{r, m}$ and send it to $r$ End for

(16) End if

(17) If all the ETRs report the old $\left(p_{r, s}^{t}, p_{r, m}^{t}\right)$ then

(18) Broadcast the packet including the "end" to all the ETRs

(19) End if

(20) If receive the updated $\left(p_{s, m}^{t}, p_{u, m}^{t}\right)$ from any VAP $s$ then

(21) Replace the old value of $\left(p_{s, m}^{t}, p_{u, m}^{t}\right)$ with its new value

(22) Else if receive the old $\left(p_{s, m}^{t}, p_{u, m}^{t}\right)$ from any VAP $s$ then

(23) Record the cumulative number of VAPs sending old values

(24) End if

(25) If all the VAPs report $\left(p_{s, m}^{t}, p_{u, m}^{t}\right)$ and there is a new value then

(26) For each VAP $s$ do compute $\varphi_{s, m}$ and send it to $s$ End for

(27) End if

(28) If all the VAPs report the old $\left(p_{s, m}^{t}, p_{u, m}^{t}\right)$ then

(29) Broadcast the packet including the "end" to all the VAPs

(32)

(35)

If $\mu_{m}\left(p_{m}^{t}, P_{r}, P_{s}\right)>\mu$ then $\left\{\mu=\mu_{m}\left(p_{m}^{t}, P_{r}, P_{s}\right) ; p_{m}^{t}=p_{m}^{t}-\varepsilon\right\}$ Else break

End if

go to (2)

End if

End while

(36) Keep energy emission at $p_{m}^{t}$

Algorithm 6: MBS-level game.

TABLE 2: Simulation parameters.

\begin{tabular}{lcc}
\hline Symbol & Description & Value \\
\hline$p_{m}^{\max }$ & Maximum transmitting power for the MBS & $40 \mathrm{dBm}$ \\
$p_{u}^{\max }$ & Maximum transmitting power for UEs, including MTRs and VAPs & $26 \mathrm{dBm}$ \\
$\mathrm{PL}_{\text {ref }}$ & Path loss at the reference distance & $30 \mathrm{~dB}$ \\
$\psi$ & Body shadowing loss margin & $15 \mathrm{~dB}$ \\
$\varphi$ & Path loss exponent & 2.3 \\
$\eta$ & Power conversion efficiency factor & 0.8 \\
$T$ & Frame length & $100 \mu \mathrm{s}$ \\
$c_{b}$ & Traffic unit price & $4.5 \mathrm{cents} / \mathrm{Mbit}$ \\
$c_{e}$ & Energy unit price & $9 \mathrm{cents} / \mathrm{KWH}$ \\
$W$ & Transmission bandwidth for each UE \\
$W_{r}$ & Transmission bandwidth for ETRs & $1 \mathrm{MHz}$ \\
$W_{s}$ & Transmission bandwidth for VAPs & $6 \mathrm{MHz}$ \\
\hline
\end{tabular}


slot parameters (i.e., $\alpha, \beta, \delta, \tau$, and $\varepsilon$ ). For any UE $u$, we set a time allocation strategy for $\varepsilon_{u}$ as follows:

$$
\varepsilon_{u}=\frac{1}{\left|U_{s}\right|} \text {. }
$$

For any ETR $r$, the adaptive time allocation for $\tau_{r}$ is determined by the following formula:

$$
\tau_{r}=\frac{T_{r}^{\max }}{\sum_{r^{\prime} \in\left\{1,2, \ldots,\left|R_{s}\right|\right\}} T_{r^{\prime}}^{\max }} .
$$

Also, the fixed time allocation for $\tau_{r}$ is determined by the following formula:

$$
\tau_{r}=\frac{1}{\left|R_{s}\right|}
$$

Our simulation metrics include average data rate for ETRs, average data rate for VAPs, and average data rate relayed by VAPs for associated UE pieces. Here, the first metric is denoted as $\mathrm{DR}_{\mathrm{av}, r}$ and estimated by the following formulas.

$$
\begin{aligned}
\mu_{r}= & T_{r}^{\max } \cdot \varphi_{r, m} \cdot W_{r} \\
& \cdot \log _{2}\left(1+\frac{\left(p_{r}^{\max }-p_{r, s}^{t}\right) \cdot g_{r, m}}{\sigma^{2}}\right) \\
N_{r}= & \left\lceil\frac{T_{r}^{\max }}{\tau_{r} \cdot(1-\beta) \cdot T}\right] \\
\mathrm{DR}_{r}= & \frac{\mu_{r}}{N_{r} \cdot T \cdot \varphi_{r, m} \cdot W_{r}} \\
\mathrm{DR}_{\mathrm{av}, r}= & \frac{\sum_{s \in S_{m}} \sum_{r \in R_{s}} \mathrm{DR}_{r}}{\left|S_{m}\right| \cdot\left|R_{s}\right|} .
\end{aligned}
$$

The second metric is denoted as $\mathrm{DR}_{\mathrm{av}, s}$ and estimated by the following formulas:

$$
\begin{aligned}
\mu_{s}= & T_{r}^{\max } \cdot \varphi_{s, m} \cdot W_{s} \\
& \cdot \log _{2}\left(1+\frac{\left(p_{s}^{\max }-p_{s, m}^{t}\right) \cdot g_{s, m}}{\sigma^{2}}\right) \\
N_{s}= & \left\lceil\frac{T_{s}^{\max }}{(1-\delta) \cdot \alpha \cdot \beta \cdot T}\right] \\
\mathrm{DR}_{s}= & \frac{\mu_{s}}{N_{s} \cdot T \cdot \varphi_{s, m} \cdot W_{s}} \\
\mathrm{DR}_{\mathrm{av}, s}= & \frac{\sum_{s \in S_{m}} \mathrm{DR}_{s}}{\left|S_{m}\right|} .
\end{aligned}
$$

The third metric is denoted as $\mathrm{DR}_{\mathrm{av}, u}$ and estimated by the following formulas:

$$
\begin{aligned}
\gamma_{u, s}^{\max } & =\frac{p_{u}^{\max } \cdot g_{u, s}}{\sigma^{2}} \\
\gamma_{t h} & =-2 \ln \text { BER }
\end{aligned}
$$

$$
\begin{aligned}
\mu_{u}= & \varepsilon_{u} \cdot(1-\beta) \cdot T \cdot W \\
& \cdot \log _{2}\left(1+\min \left\{\gamma_{u, s}^{\max }, \gamma_{t h}\right\}\right) \\
\mu_{s}^{\prime}= & \delta \cdot \alpha \cdot \beta \cdot T \cdot W \cdot \log _{2}\left(1+\frac{p_{s, m}^{t} \cdot g_{s, m}}{\sigma^{2}}\right) \\
N_{s}^{\prime}= & {\left[\frac{T_{s}^{\max }}{\delta \cdot \alpha \cdot \beta \cdot T}\right] } \\
\mathrm{DR}_{u}= & \left\{\begin{array}{l}
\frac{\sum_{u \in U_{s}} \mu_{u}}{N^{\prime} \cdot T \cdot W} \\
\log _{2}\left(1+\frac{p_{s, m}^{t} \cdot g_{s, m}}{\sigma^{2}}\right) \quad \mu_{u \in U_{s}}>\mu_{s}^{\prime}
\end{array}\right. \\
\mathrm{DR}_{\mathrm{av}, u}= & \frac{\sum_{s \in S_{m}} \sum_{u \in U_{s}} \mathrm{DR}_{u}}{\left|S_{m}\right| \cdot\left|U_{s}\right|} .
\end{aligned}
$$

In (32), BER is bit error ratio and takes different values (e.g., $10^{-8}$ ). When the set number of UE pieces (i.e., $\left|U_{s}\right|$ ) must meet the following relational expression, the relational expressions in (32) are true.

$$
\begin{gathered}
\sum_{u \in U_{s}} \frac{(1-\beta) \cdot \log _{2}\left(1+\min \left\{\gamma_{u, s}^{\max }, \gamma_{t h}\right\}\right)}{\left|U_{s}\right|} \\
<\left\lceil\frac{T_{s}^{\max }}{T}\right] \cdot \log _{2}\left(1+\frac{p_{s, m}^{t} \cdot g_{s, m}}{\sigma^{2}}\right) .
\end{gathered}
$$

In this paper, the meaning of $\lceil X\rceil$ is the integer that is not less than $X$.

4.3. Simulation Results and Analysis. Firstly, we consider the two groups of simulations, which adopt adaptive time allocation for $\tau_{r}$ (i.e., formula (28)). In the first group of simulations, the channel noise power $\sigma^{2}$ at all the receivingends is fixed as $-90 \mathrm{dBm} / \mathrm{MHz}$, and the parameter $\varepsilon$ is set as $0.1 \%$. We evaluate the performance in terms of the three metrics under the change of average UE battery capacity level (i.e., the value of $X$ ), as shown in Figure 6.

From Figures 6(a) and 6(b), we see that the change of average UE battery capacity has little impact on the average data rates of ETRs and VAPs. The main reason can be found from formula (30) and formula (31). Without loss of generality, we take formula (30) as an example, where, on the one hand, the continuous emission time $T_{r}{ }^{\max }$ gets longer with increase of average UE battery capacity, and thus the amount of emitted data is larger during $T_{r}{ }^{\max }$; on the other hand, the number of time frames proportionately increases with the increase of $T_{r}$ max . Therefore, according to formula (30), the average data rate for ETRs has been hardly changed.

As shown in Figure 6(c), the change of average data rate for associated UE pieces with increase of average UE battery capacity is different from those of ETRs and VAPs. For the associated UE pieces, when the data generated in a time frame is greater than the forwarding amount of their associating VAP in the same time frame, more time frames 


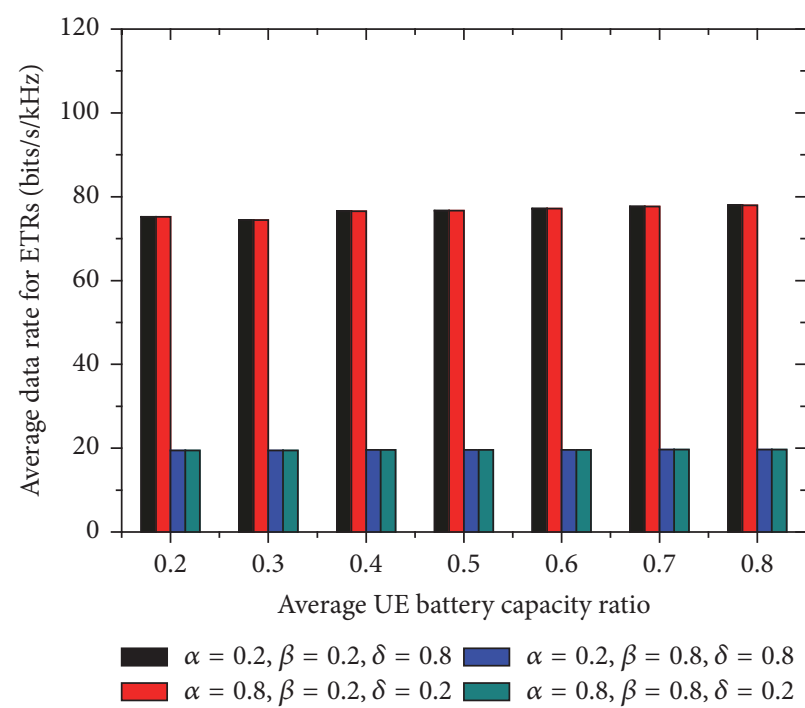

(a) ETR data rate versus UE battery capacity

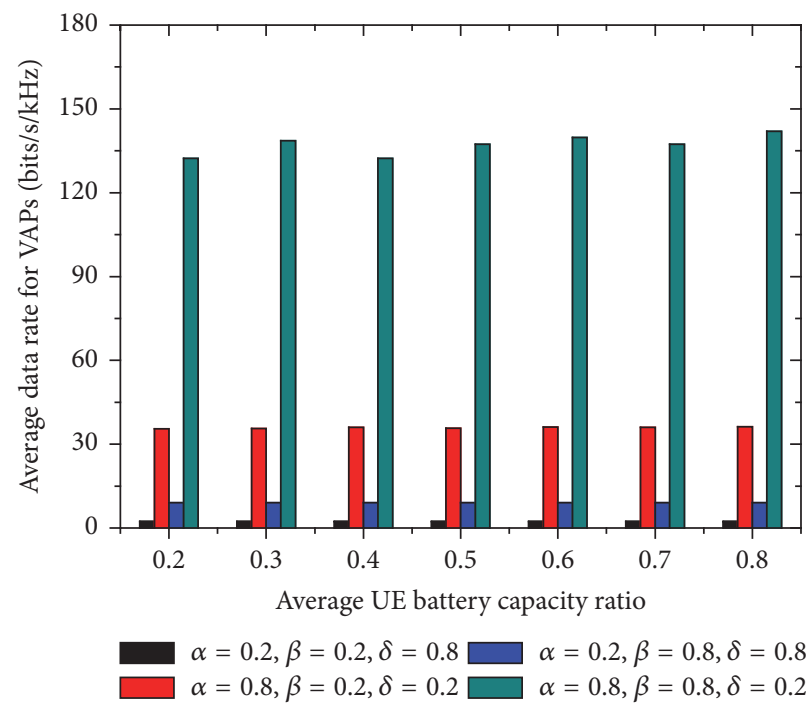

(b) VAP data rate versus UE battery capacity

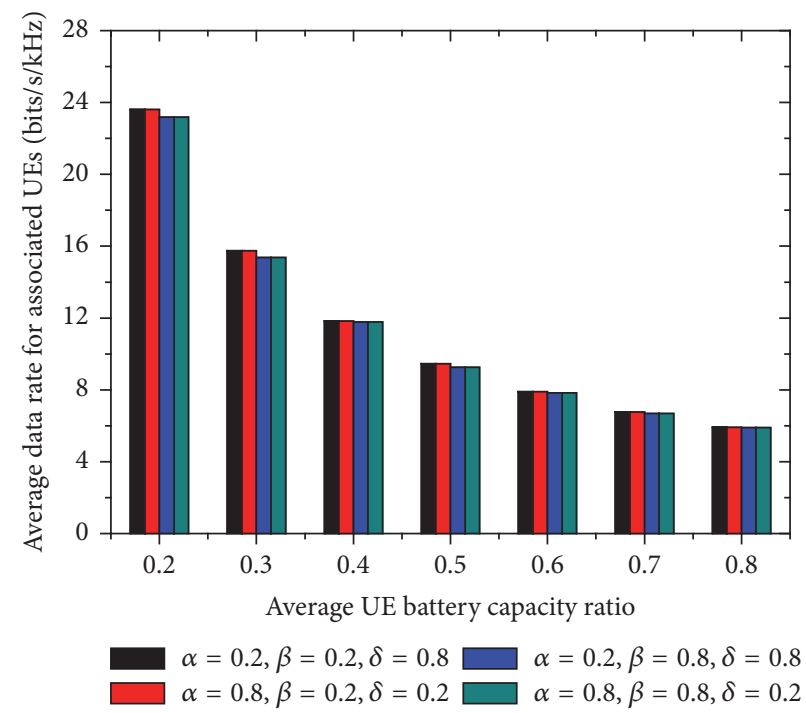

(c) The data rate of associated UE versus UE capacity

FIGURE 6: Average date rate versus UE battery capacity under adaptive time allocation.

are required to complete the forwarding task. When average UE battery capacity is higher, the continuous emission time $T_{s}{ }^{\max }$ gets longer and thus the number of time frames is larger. Therefore, according to formula (32), the average data rate for associated UE pieces decreases with increase of average UE battery capacity, since it is limited to the forwarding capacity of the associating VAP.

In addition, the time slot parameters (i.e., $\alpha, \beta$, and $\delta$ ) show the different effects on the three data rates. For the average data rate of ETRs, smaller value for $\beta$ has more positive effect on its improvement. This is because, in a given time frame, any ETR will get more time to transmit its own data when $\beta$ takes smaller value (see Figure 4(a)). For the average data rate of VAPs, when the values for both $\alpha$ and $\beta$ are very large, this metric can be significantly improved. Even though the value for $\beta$ is very small, this metric is relatively good when the value for $\alpha$ is large enough and the value for $\delta$ is also small enough. These phenomena can also be explained by the amount of transmitting time allocated in the same time frame (see Figure 4(b)).

From Figure 6(c), we find that the time slot parameters have little impact on the average data rate for associated UE pieces. As explained in the previous text, the average data rate for associated UE pieces is limited to the forwarding capacity of the associating VAP, where the forwarding capacity of the associating VAP is unaffected by the time slot parameters according to formula (32).

In the second group of simulations, the average UE battery capacity level for all UE pieces is fixed (e.g., $X$ is fixed as $50 \%$ ), and the parameter $\varepsilon$ is set as $0.1 \%$. We evaluate the performance in terms of the three metrics under the change of channel noise power as shown in Figure 7. 


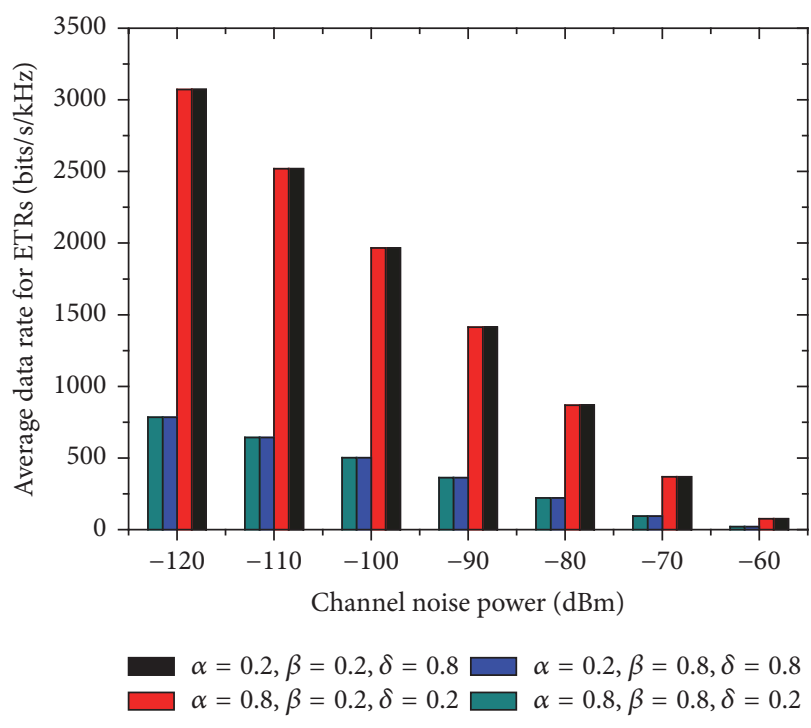

(a) ETR data rate versus channel noise power

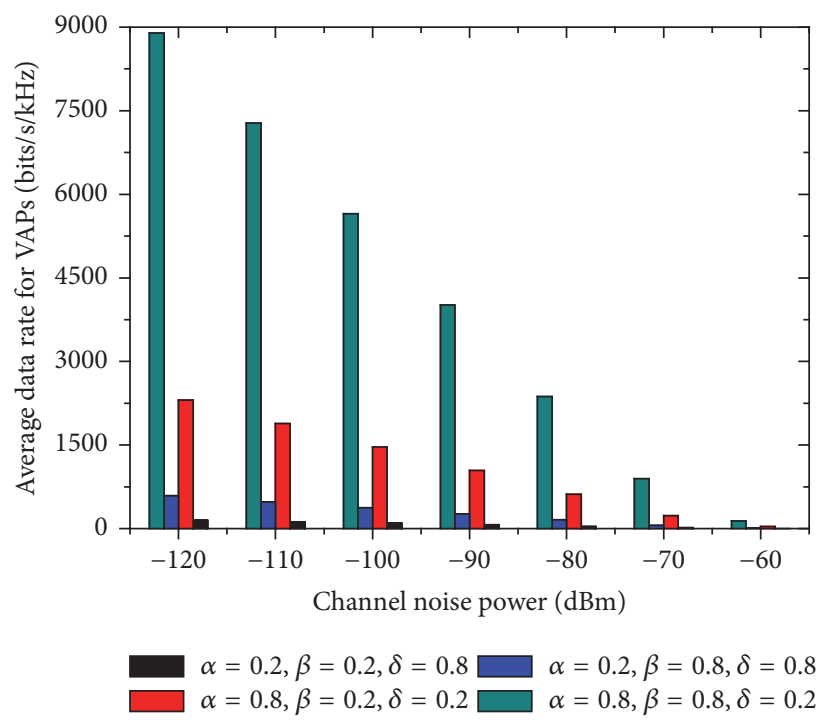

(b) VAP data rate versus channel noise power

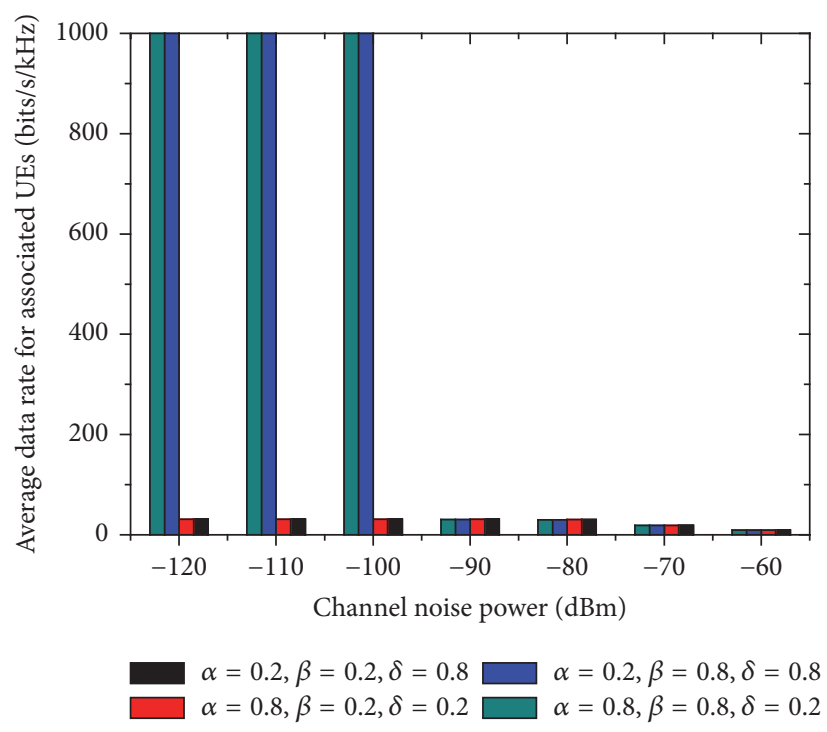

(c) The data rate of associated UE versus channel noise power

FIGURE 7: Average date rate versus channel noise power under adaptive time allocation.

From Figure 7, we see that, with increase of channel noise power, the three data rates show a downward trend. This is because a larger channel noise power leads to a higher bit error ratio on a receiving end when the corresponding transmitting power is basically unchanged.

Unlike the variation trend in Figures $7(a)$ and 7(b), there is a sudden change for the schemes with larger value of $\beta$ in Figure 7(c) when channel noise power is changed from -100 to $-90 \mathrm{dBm} / \mathrm{MHz}$. This can be explained by formula (32). That is, when channel noise power is small enough, (e.g., less than $-90 \mathrm{dBm} / \mathrm{MHz}$ ), as stated in the previous text, the forwarding amount of a VAP in a time frame is greater than the data generated by its associating UE pieces in same time frame. Therefore, it can complete the forwarding task in a single time frame and thus obviously improve the average data rate for associated UE pieces.

However, for the schemes with very small value of $\beta$ in Figure 7(c), the change of channel noise power has little impact on the average data rate for associated UE pieces especially when channel noise power is less than $-70 \mathrm{dBm} / \mathrm{MHz}$. This is because, in this situation, the forwarding amount of a VAP in a time frame is usually much lower than the data generated by its associating UE pieces in same time frame, and thus almost all the VAPs need more time frames to complete forwarding task. Therefore, it is not too unlikely that the change of noise power leads to the sudden change of data rate. In addition, with respect to the time slot parameters of impact on the three data rates, the same 


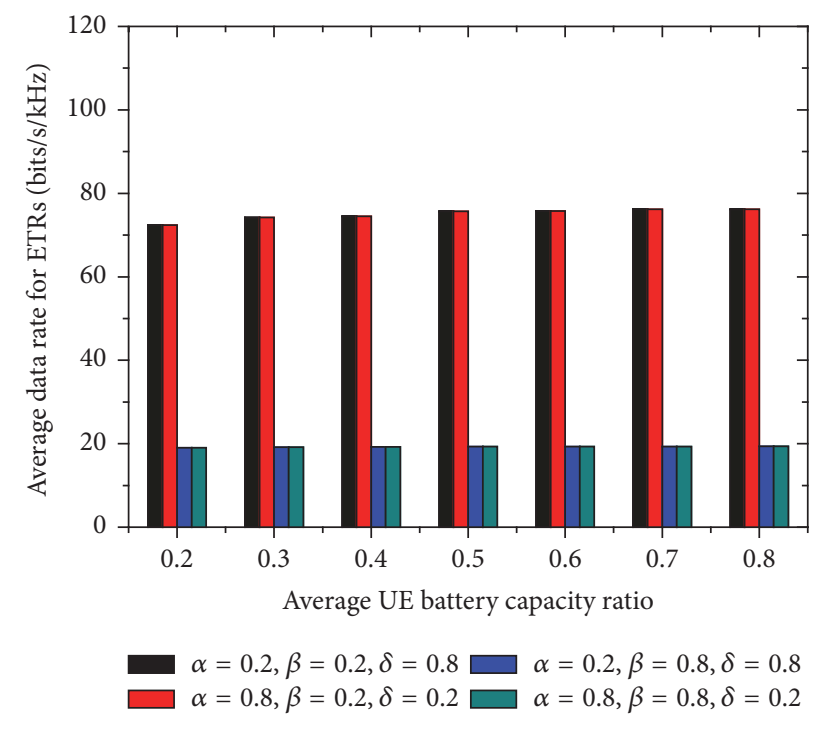

FIGURE 8: Average date rate versus UE battery capacity under fixed time allocation.

explanation in Figure 6 can be used to explain the situation in Figure 7.

Then, our simulations use the fixed time allocation for $\tau_{r}$ (i.e., formula (29)) instead of the adaptive time allocation for $\tau_{r}$ (i.e., formula (28)), where the purpose is to compare the average data rates of ETRs under the two types of time allocation schemes, since the average data rates of VAPs and associated UE pieces are independent of the parameter $\tau_{r}$. The other configuration of simulation parameters is the same as those in the above two groups of simulations. The simulation results are shown in Figures 8 and 9.

When comparing Figure 8 with Figure 6(a), we observe that the difference between the two time allocation schemes is very small. It may be that the advantage of adaptive time allocation is not obvious due to the adoption of relatively short time frame. Also, when comparing Figure 9 with Figure 7(a), we can find similar result.

Finally, we evaluate the game convergence rates of ETR, VAP, and MBS under the variation of step size (i.e., the parameter $\varepsilon$ ) through the simulation result shown in Figure 10. Also, we evaluate the average date rates of ETR, VAP, and associated UE pieces under the variation of step size through the simulation result shown in Figure 11.

From Figure 10, we see that a larger step size leads to a faster convergence. The reason behind this phenomenon is straightforward from the description of Algorithm 4-6. Meanwhile, from Figure 11, we find that the variation of step size has little impact on the average date rate. Therefore, the step size can be appropriately increased to speed up the convergence of the proposed game scheme.

In addition, Figure 10 shows the game rounds of a MBS are obviously less than those of ETRs and VAPs. From the description of Algorithm 6, we can easily find the reason. Also, from Figure 11, we see that a VAP is highly efficient in terms of its own data transmission. Based on balance between contribution and return in terms of data transmission,

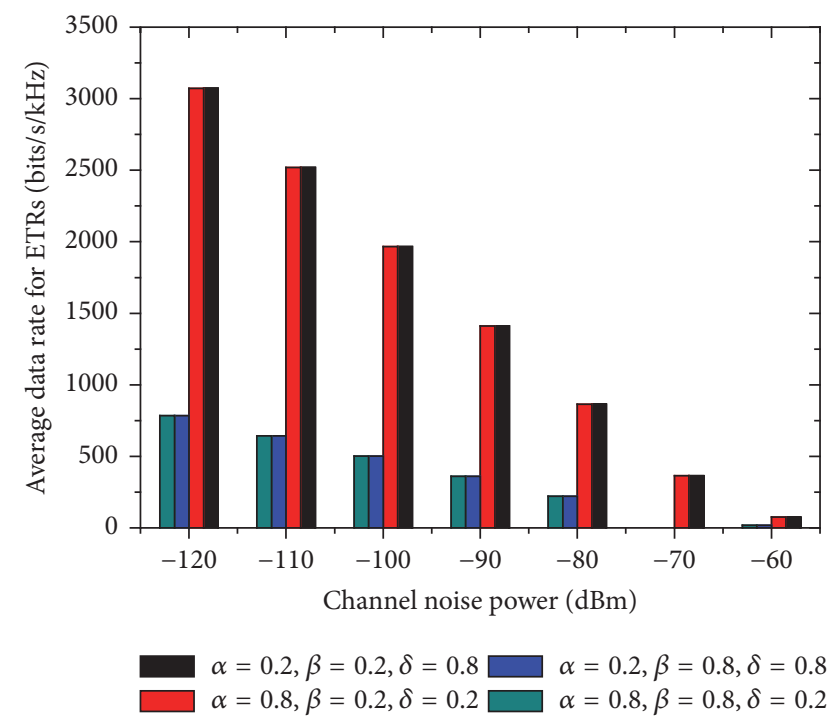

FIGURE 9: Average data rate versus channel noise power under fixed time allocation.

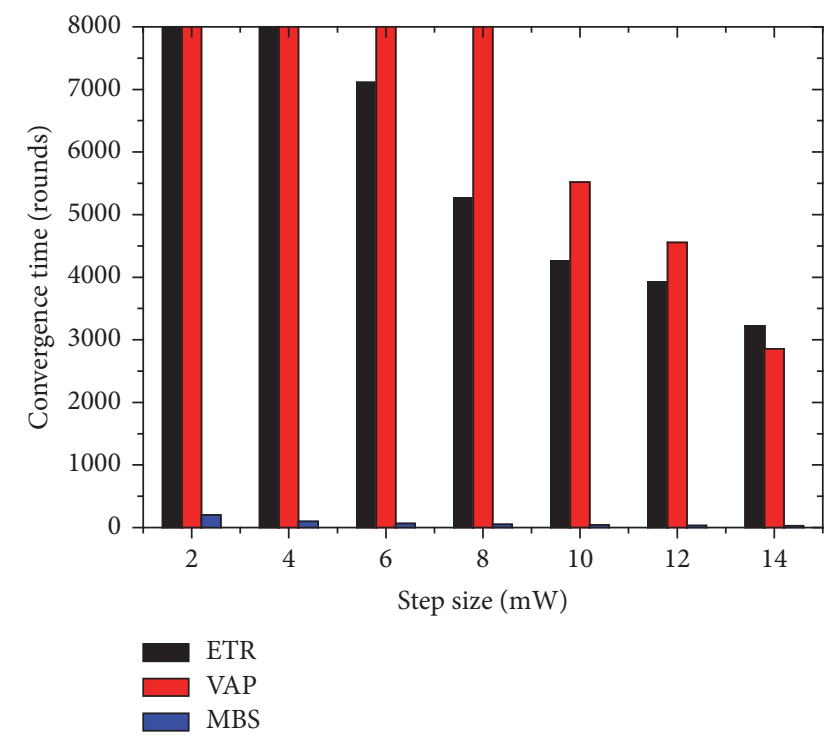

FIgURE 10: Average convergence time versus step size.

network operators can consider allocating fewer frequency bands or time slots to VAPs for their Internet access.

\section{Conclusion}

This paper explores a charging power decision and distributed transmission power splitting problem based on game theory for constructing virtual cellular coverage area in overlay cellular networks. To characterize rational behaviors of wireless terminal users, we formulate a noncooperative game for the considered system, where each terminal user is modeled as a strategic player that aims to maximize its own data rate under the constraint for its maximum transmission power. 


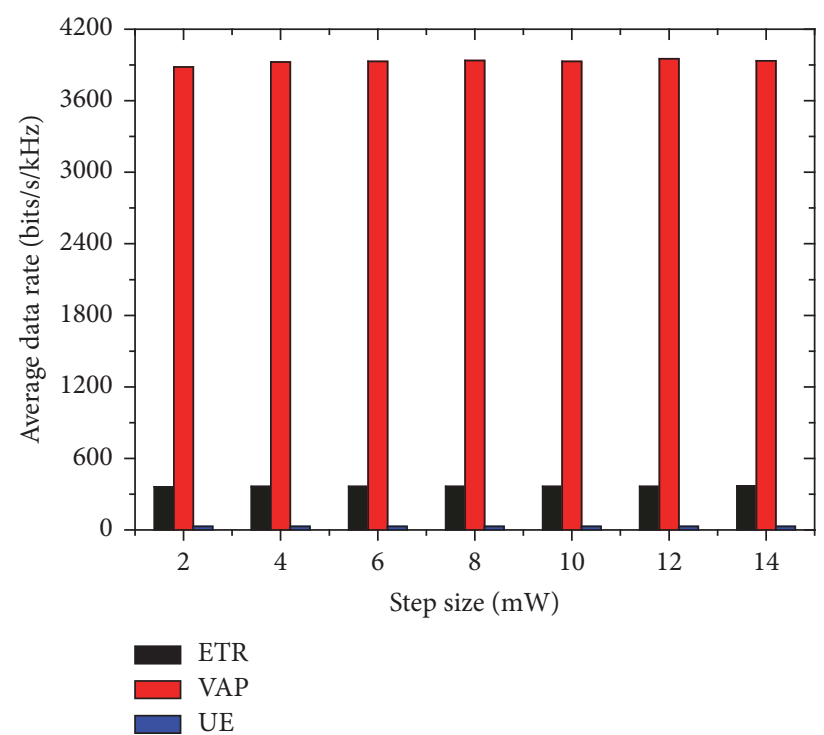

FIgURE 11: Average data rate versus step size.

Theoretical analysis proves the convergence of the proposed game scheme. Simulation results indicate that, under the certain conditions, the game-theoretic charging power decision and transmission power splitting mechanism can speed up the convergence on the premise of QoS requirement in terms of average date rate. However, we do not consider the optimizing problem for time allocation, since we focus on the design and analysis of game scheme for virtual cellular coverage area construction. Therefore, we plan to address it in our future work.

\section{Notations Used in Our Work}

$S_{m}$ : The set of VAPs

$R_{s}$ : The set of ETRs associated with a VAP (e.g., $s$ )

$U_{s}$ : The set of UEs associated with a VAP (e.g., $s)$

$|\cdot|$ : The number of members in a set (e.g., $\left|S_{m}\right|$ is the number of the VAPs in $S_{m}$ )

$T: \quad$ The time frame for communication

$\beta$ : The fraction of energy transfer time in $T$ time

$\alpha$ : The fraction of energy transfer time from the MBS to ETRs in $\beta \cdot T$ time

$\delta$ : The fraction of information transmission time from a VAP to MBS in $\alpha \cdot \beta \cdot T$ time for the data received by the VAP from its associating UEs

$\tau_{r}:$ The ratio of time when an ETR transfers energy to its associating VAP

$\varepsilon_{u}$ : The ratio of time when UE transmits its data to its associating VAP

$p_{i, j}^{t}:$ The transmission power at sender $i$ in link $i \rightarrow j$

$p_{i, j}^{r}:$ The receiving power at receiver $j$ in link $i \rightarrow j$ $\eta_{i, j}:$
$h_{i, j}:$
$\varphi:$
$\mathrm{PL}_{\mathrm{ref}}:$
$\psi_{i, j}:$
$\beta_{i, j}:$
$d_{i, j}:$
$W_{:}:$
$W_{r}:$
$W_{s}:$
$\sigma^{2}:$
$b_{i, j}:$

$p_{r}^{\max }$ :

$p_{s}^{\max }:$

$E_{r}:$

$T_{r}^{\max }$ :

$T_{s}^{\text {max }}$ :

$\mu_{r}\left(P_{r}\right)$ :

$\mu_{s}\left(P_{s}\right):$

$\mu_{m}\left(p_{m}^{t}, P_{r}, P_{s}\right)$ :

$\varphi_{r, m}$ :

$\varphi_{s, m}:$

$\emptyset_{r}, \emptyset_{s}:$

$p_{r, s}^{t *}:$

$p_{m}^{t *}:$

$e_{s}$ :

$c_{b}:$

$c_{e}$ :

$\varepsilon:$

$E_{i}$ :

$E_{\text {av }}$ :

$R_{t h}$ :

$\mathrm{DR}_{r}$ :

$\mathrm{DR}_{\mathrm{av}, r}$ :

$\mathrm{DR}_{s}$ :

$\mathrm{DR}_{\mathrm{av}, s}$ :

$\mathrm{DR}_{u}$ :

$\mathrm{DR}_{\mathrm{av}, u}$ :
The power conversion efficiency factor from sender $i$ to receiver $j$

The channel power gain from sender $i$ to receiver $j$

Path loss exponent

The path loss in $\mathrm{dB}$ at the reference distance

The body shadowing loss margin between sender $i$ and receiver $j$ in $\mathrm{dB}$

The small-scale fading power gain between sender $i$ and receiver $j$

The distance between sender $i$ and receiver j

Transmission bandwidth for each UE

Transmission bandwidth for ETRs

Transmission bandwidth for VAPs

Noise power

The information decoding rate in link $i \rightarrow j$

The maximum transmission power of ETR $r$

The maximum transmission power of VAP

The battery energy storage of ETR $r$

The continuous emission time of ETR $r$

The continuous emission time of VAP $s$

The utility of ETR $r$

The utility of VAP $s$

The utility of MBS $m$

The fraction of bandwidth for ETR $r$ sending its data to MBS $m$

The fraction of bandwidth for VAP $s$ sending its data to MBS $m$

The contribution from a fictitious selfless node

The ETR $r$ 's optimal charging power to

VAP $s$

The MBS m's optimal charging power to

ETRs

The harvesting energy of VAP $s$ from the $\left|R_{s}\right|$ ETRs

Traffic price per bit

Energy price per joule

The step length for power adjustment

The initial battery capacity of UE $i$

The average initial battery capacity

The distance from reference points to MBS

The data rate for ETR $r$

The average data rate for ETRs

The data rate for VAP $s$

The average data rate for VAPs

The data rate for UE $u$ associated with a

VAP

The average data rate relayed by a VAP for its associated UEs.

\section{Conflicts of Interest}

The authors declare that there are no conflicts of interest regarding the publication of this article. 


\section{Acknowledgments}

This work was supported in part by the National Natural Science Foundation of China (61272494, 61379110).

\section{References}

[1] M. Wu, Y. Wu, X. Liu, M. Ma, A. Liu, and M. Zhao, "Learningbased synchronous approach from forwarding nodes to reduce the delay for Industrial Internet of Things," EURASIP Journal on Wireless Communications and Networking, vol. 2018, no. 10, 2018.

[2] M. Huang, A. Liu, T. Wang, and C. Huang, "Green data gathering under delay differentiated services constraint for internet of things," Wireless Communications and Mobile Computing, vol. 2018, Article ID 9715428, 23 pages, 2018.

[3] J. Tang, A. Liu, M. Zhao, and T. Wang, "An Aggregate Signature Based Trust Routing for Data Gathering in Sensor Networks," Security and Communication Networks, vol. 2018, Article ID 6328504, 30 pages, 2018.

[4] A. Osseiran, V. Braun, T. Hidekazu et al., "The foundation of the mobile and wireless communications system for 2020 and beyond: Challenges, Enablers and technology solutions," in Proceedings of the 2013 IEEE 77th Vehicular Technology Conference, VTC Spring 2013, IEEE, Dresden, Germany, 2013.

[5] K. Zhou, J. Gui, and N. Xiong, "Improving cellular downlink throughput by multi-hop relay-assisted outband D2D communications," EURASIP Journal on Wireless Communications and Networking, vol. 2017, no. 209, 2017.

[6] J. Gui and J. Deng, "Multi-hop Relay-Aided Underlay D2D Communications for Improving Cellular Coverage Quality," IEEE Access, vol. 6, pp. 14318-14338, 2018.

[7] Cisco Visual Networking Index: Forecast and Methodology, 2016-2021, CISCO White Paper, 2017.

[8] J. Gui and K. Zhou, "Flexible adjustments between energy and capacity for topology control in heterogeneous wireless multihop networks," Journal of Network and Systems Management, vol. 24, no. 4, pp. 789-812, 2016.

[9] J. Gui and J. Deng, "A topology control approach reducing construction cost for lossy wireless sensor networks," Wireless Personal Communications, vol. 95, no. 3, pp. 2173-2202, 2017.

[10] D. Mishra, De. Swades, S. Jana, S. Basagni, K. Chowdhury, and W. Heinzelman, "Smart RF energy harvesting communications: Challenges and opportunities," IEEE Communications Magazine, vol. 53, no. 4, pp. 70-77, 2015.

[11] S. Bi, C. K. Ho, and R. Zhang, "Wireless powered communication: opportunities and challenges," IEEE Communications Magazine, vol. 53, no. 4, pp. 117-125, 2015.

[12] K. Huang and X. Zhou, "Cutting the last wires for mobile communications by microwave power transfer," IEEE Communications Magazine, vol. 53, no. 6, pp. 86-93, 2015.

[13] H. Ju and R. Zhang, "Throughput maximization in wireless powered communication networks," IEEE Transactions on Wireless Communications, vol. 13, no. 1, pp. 418-428, 2014.

[14] H. Ju and R. Zhang, "User cooperation in wireless powered communication networks," in Proceedings of the IEEE Global Communications Conference (GLOBECOM '14), pp. 1430-1435, IEEE, Austin, Tex, USA, December 2014.

[15] L. Liu, R. Zhang, and K. C. Chua, "Multi-antenna wireless pow ered communication with energy beamforming," IEEE Transactions on Communications, vol. 62, no. 12, pp. 4349-4361, 2014.
[16] G. Yang, C. K. Ho, R. Zhang, and Y. L. Guan, "Throughput optimization for massive MIMO systems powered by wireless energy transfer," IEEE Journal on Selected Areas in Communications, vol. 33, no. 8, pp. 1640-1650, 2015.

[17] X. Kang, C. K. Ho, and S. Sun, "Full-duplex wireless-powered communication network with energy causality," IEEE Transactions on Wireless Communications, vol. 14, no. 10, pp. 5539-5551, 2015.

[18] H. Ju and R. Zhang, "Optimal resource allocation in full-duplex wireless-powered communication network," IEEE Transactions on Communications, vol. 62, no. 10, pp. 3528-3540, 2014.

[19] D. W. K. Ng, E. S. Lo, and R. Schober, "Wireless information and power transfer: energy efficiency optimization in OFDMA systems," IEEE Transactions on Wireless Communications, vol. 12, no. 12, pp. 6352-6370, 2013.

[20] K. Yamazaki, Y. Sugiyama, Y. Kawahara, S. Saruwatari, and T. Watanabe, "Preliminary evaluation of simultaneous data and power transmission in the same frequency channel," in Proceedings of the 2015 IEEE Wireless Communications and Networking Conference, (WCNC), pp. 1237-1242, New Orleans, LA, USA, 2015.

[21] D. Altinel and G. Karabulut Kurt, "Energy Harvesting from Multiple RF Sources in Wireless Fading Channels," IEEE Transactions on Vehicular Technology, vol. 65, no. 11, pp. 8854-8864, 2016.

[22] J. C. Kwan and A. O. Fapojuwo, "Radio Frequency Energy Harvesting and Data Rate Optimization in Wireless Information and Power Transfer Sensor Networks," IEEE Sensors Journal, vol. 17, no. 15, pp. 4862-4874, 2017.

[23] L. Xiang, J. Luo, K. Han, and G. Shi, "Fueling wireless networks perpetually: A case of multi-hop wireless power distribution," in Proceedings of the IEEE 24th Annual International Symposium on Personal, Indoor, and Mobile Radio Communications, (PIMRC), pp. 1994-1999, UK, 2013.

[24] T. Rault, A. Bouabdallah, and Y. Challal, "Multi-hop wireless charging optimization in low-power networks," in Proceedings of the IEEE Global Communications Conference, (GLOBECOM), pp. 462-467, IEEE, Atlanta, GA, USA, 2013.

[25] D. Fudenberg and J. Tirole, Game Theory, MIT Press, Cambridge, Mass, USA, 1993.

[26] D. Monderer and L. S. Shapley, "Potential games," Games and Economic Behavior, vol. 14, no. 1, pp. 124-143, 1996.

[27] F. Ma, X. Liu, A. Liu, M. Zhao, C. Huang, and T. Wang, "A Time and Location Correlation Incentive Scheme for Deep Data Gathering in Crowdsourcing Networks," Wireless Communications and Mobile Computing, vol. 2018, Article ID 8052620, 22 pages, 2018.

[28] J. Gui, Y. Lu, X. Deng, and A. Liu, "Flexible resource allocation adaptive to communication strategy selection for cellular clients using Stackelberg game," Ad Hoc Networks, vol. 66, pp. 64-84, 2017.

[29] J. Gui, L. Hui, and N. Xiong, "A game-based localized multiobjective topology control scheme in heterogeneous wireless networks," IEEE Access, vol. 5, no. 1, pp. 2396-2416, 2017.

[30] H. Chen, Y. Li, Y. Jiang, Y. Ma, and B. Vucetic, "Distributed power splitting for SWIPT in relay interference channels using game theory," IEEE Transactions on Wireless Communications, vol. 14, no. 1, pp. 410-420, 2015.

[31] Y. Ma, H. H. Chen, Z. Lin, B. Vucetic, and X. Li, "Spectrum sharing in RF-powered cognitive radio networks using game theory," in Proceedings of the 26th IEEE Annual International 
Symposium on Personal, Indoor, and Mobile Radio Communications, PIMRC, pp. 992-996, IEEE, Hong Kong, China, 2015.

[32] H. H. Chen, Y. Li, Z. Han, and B. Vucetic, "A stackelberg gamebased energy trading scheme for power beacon-assisted wireless-powered communication," in Proceedings of the 40th IEEE International Conference on Acoustics, Speech, and Signal Processing, (ICASSP'15), pp. 3177-3181, IEEE, Brisbane, Australia, 2014.

[33] Y. Ma, H. Chen, Z. Lin, Y. Li, and B. Vucetic, "Distributed and optimal resource allocation for power beacon-assisted wirelesspowered communications," IEEE Transactions on Communications, vol. 63, no. 10, pp. 3569-3583, 2015.

[34] H. Chen, Y. Ma, Z. Lin, Y. Li, and B. Vucetic, "Distributed Power Control in Interference Channels with QoS Constraints and RF Energy Harvesting: A Game-Theoretic Approach," IEEE Transactions on Vehicular Technology, vol. 65, no. 12, pp. 1006310069, 2016.

[35] Z. Hou, H. Chen, Y. Li, and B. Vucetic, "Incentive Mechanism Design for Wireless Energy Harvesting-Based Internet of Things," IEEE Internet of Things Journal, 2017.

[36] E. Dahlman, S. Parkvall, and J. Skold, "Propagation model and channel characterization," in Communications Engineering Desk Reference, pp. 247-256, Academic Press, Oxford, UK, 2009. 


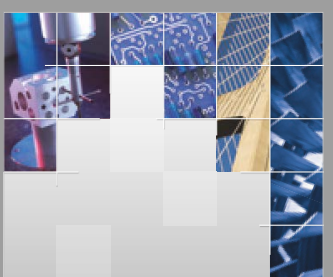

\section{Enfincering}
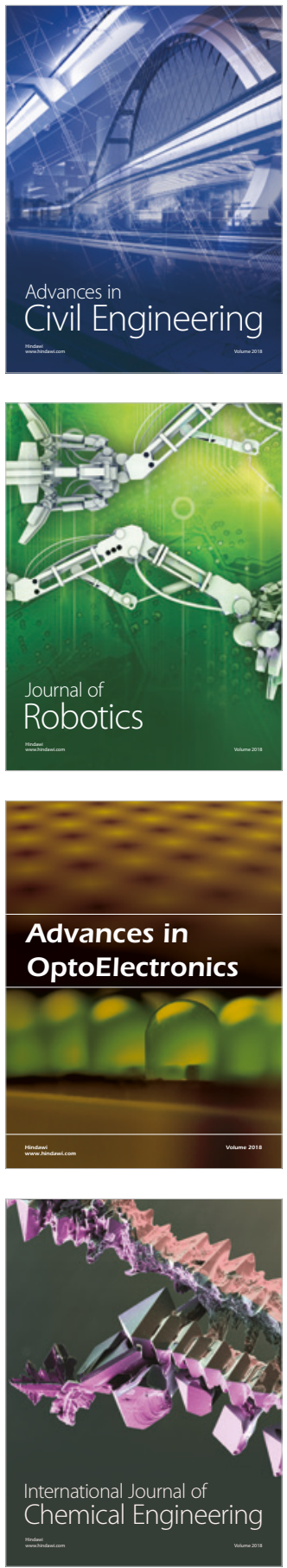

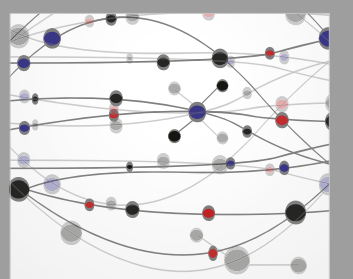

\section{Rotating \\ Machinery}

The Scientific World Journal

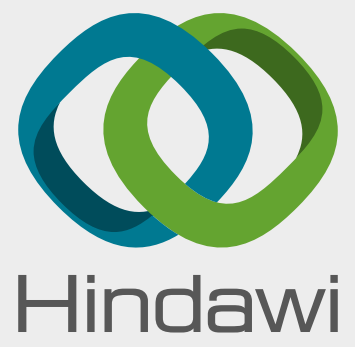

Submit your manuscripts at

www.hindawi.com
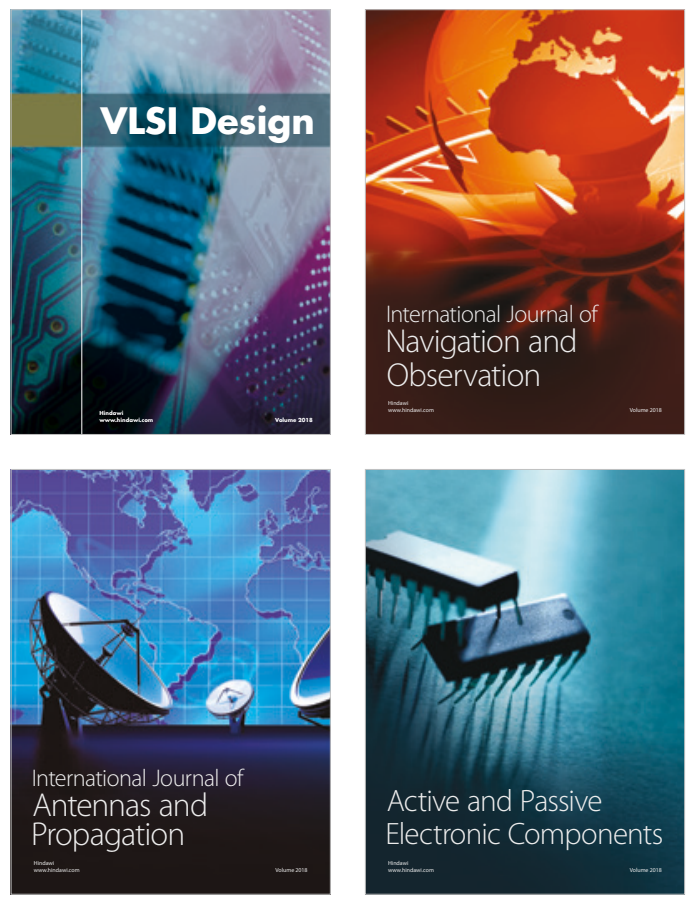
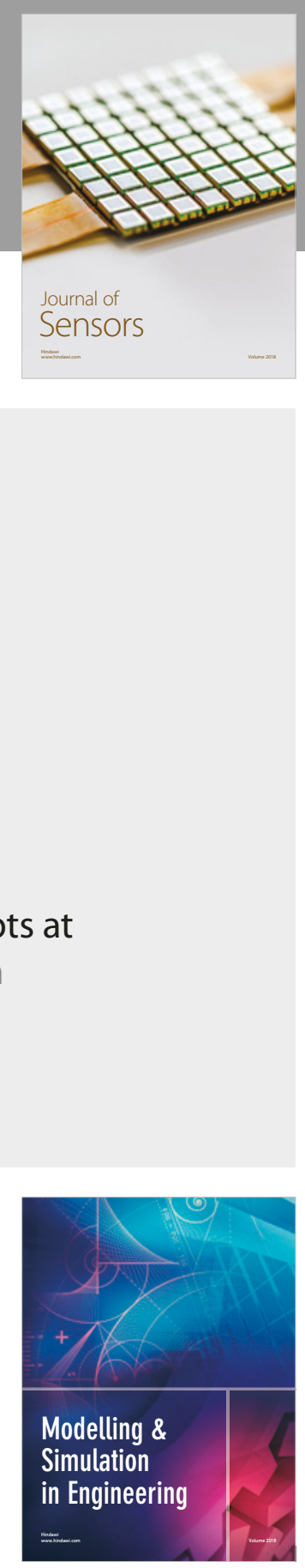

\section{Advances \\ Multimedia}
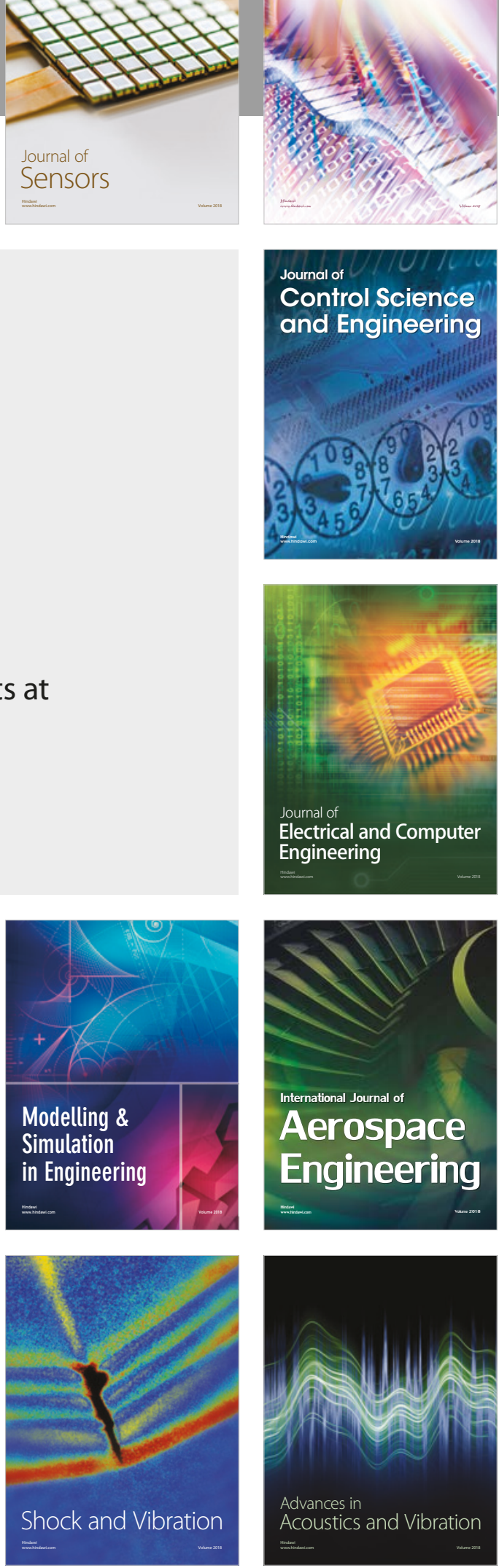